\title{
Molecular Docking, Synthesis and Biological Evaluation of Some Novel 2-Substituted-3-allyl-4(3H)-quinazolinone Derivatives as Anticonvulsant Agents
}

\author{
Hamada S Abulkhair ${ }^{1}$, Kamal M El-Gamal ${ }^{1,2}$, Khaled El-Adl ${ }^{3 *}$ and Mohamed F FadI ${ }^{4}$ \\ ${ }^{1}$ Pharmaceutical Organic Chemistry Department, Faculty of Pharmacy, Al-Azhar University, Nasr City 11884, Cairo, Egypt \\ ${ }^{2}$ Pharmaceutical Organic Chemistry Department, Faculty of Pharmacy and Pharmaceutical Industries, Delta University, Mansoura, Egypt \\ ${ }^{3}$ Pharmaceutical Chemistry Department, Faculty of Pharmacy, Al-Azhar University, Nasr City 11884, Cairo, Egypt \\ ${ }^{4}$ Pharmacology and Toxicology Department, Faculty of Pharmacy, Al-Azhar University, Nasr City 11884, Cairo, Egypt
}

\begin{abstract}
A new series of 2-substituted-3-allyl-4(3H)-quinazolinone derivatives $\left(4_{a-e}-8_{a-d}\right)$ were synthesized and evaluated for their anticonvulsant activity against pentylenetetrazole (PTZ)-induced seizures and maximal electroshock test in mice and compared with the reference drugs methaqualone and sodium valproate. The neurotoxicity was assessed using rotarod test. The molecular modeling was performed for all synthesized compounds to predict their binding affinity towards GABA-A receptor as a proposed mode of their anticonvulsant activity. The data obtained from the molecular docking was strongly correlated with that obtained from the biological screening which revealed that; compounds $\mathbf{4}_{c}, \mathbf{4}_{b}$ and $\mathbf{4}_{d}$ showed the highest binding affinities towards GABA-A receptor and also showed the highest anticonvulsant activities in experimental mice with relatively low neurotoxicity and low toxicity in the median lethal dose test when compared with the reference drugs. The obtained results proved that the most active compounds could be useful as a model for future design, adaptation and investigation to construct more active analogs.
\end{abstract}

Keywords: Quinazolinone; Anticonvulsant; GABA-A receptor; Molecular docking

\section{Introduction}

Quinazolin-4(3H)-ones and their derivatives constitute an important class of heterocyclic compounds and are shown to have potent CNS activities such as anticonvulsant [1-6] and CNS depressant [7]. 2-Methyl-3-O-tolyl 4(3H)-quinazolinone (methaqualone) is an important landmark in the field of synthetic anticonvulsant, possessed quinazoline core which was responsible for its activity $[8,9]$. Modification of the methyl group at position 2 by some other chemical moieties yielded structural analogs with potent CNS activity [5]. The sedative-hypnotic (neurotoxicity) properties of $4(3 \mathrm{H})$-quinazolinone are well documented [10].

Many quinazoline derivatives were reported as GABA-A receptor stimulants $[2,5]$. The GABA-A receptor is an ionotropic receptor and a ligand-gated ion channel. Its endogenous ligand is $\gamma$-aminobutyric acid (GABA), the major inhibitory neurotransmitter in the central nervous system. Upon activation, the GABA-A receptor selectively conducts $\mathrm{Cl}^{-}$through its pore, resulting in hyperpolarization of the neuron. This causes an inhibitory effect on neurotransmission by diminishing the chance of a successful action potential [2]. The active site of the GABA-A receptor is the binding site for GABA and several drugs such as muscimol. The protein also contains a number of different allosteric binding sites which modulate the activity of the receptor indirectly. These allosteric sites are the targets of various other drugs, including benzodiazepines, barbiturates and ethanol [11,12]. Methaqualone is a positive allosteric GABA-A receptor modulator. It binds to allosteric sites on the GABA-A receptor complex and affects it in a positive manner, causing increased efficiency of the main site and therefore an indirect increase in $\mathrm{Cl}^{-}$conductance [11,12]. Many quinazolinones structurally related to compound methaqualone were synthesized and biologically tested for their anticonvulsant activity. None of those compounds are currently used [8]. A persistent problem encountered with these compounds arises from the fact that, nearly every derivative tested in combined neurotoxicity and anticonvulsant screenings exhibited neurotoxicity values $\left(\mathrm{TD}_{50}\right.$ 's) that are less than or only slightly higher than the effective doses $\left(\mathrm{ED}_{50}\right.$ 's) consequently, the protective index (PI) corresponding to $\left(\mathrm{TD}_{50} / \mathrm{ED}_{50}\right)$ is too low [13].

In continuation to the efforts done toward the synthesis of potential molecules as anticonvulsant agents, our aim was to synthesize new 2-substituted-3-allyl-4(3H)-quinazolinone derivatives $\left(4_{\text {a-e }}\right.$ 8 ) derivatives and evaluate their anticonvulsant potency. It was of special importance to incorporate some moieties that were reported to potentiate the anticonvulsant activity such as ketone, amide, ester, hydrazide and/or arylidene [12,14]. Substituents were selected following a second manual method of the Hansch approach to drug design suggested by Topliss [15]. Hansch approach is a procedure in which an initial small group of compounds are selected, tested and ordered according to potency.

The title compounds were designed to contain unsubstituted, monosubstituted and disubstituted aromatic ring with different kinds of halogens and other hydrophobic and hydrophilic groups of different electronic environment either electronic rich or electronic deficient groups to study SAR of these compounds and compare the differences in their anticonvulsant activity.

\section{Experimental Protocol}

\section{Chemistry}

Open capillary tubes were used to detect melting points. Pye Unicam SP 3300 spectrophotometer was used to record the IR spectra (in $\mathrm{KBr}$

*Corresponding author: Khaled El-Adl, Assistant Professor of Pharmaceutical Chemistry, Faculty of Pharmacy, Al-Azhar University, Cairo, Egypt, Tel +20224018031; E-mail: eladlkhaled74@yahoo.com

Received September 23, 2016; Accepted September 29, 2016; Published September 30, 2016

Citation: Abulkhair HS, El-Gamal KM, El-Adl K, Fadl MF (2016) Molecular Docking Synthesis and Biological Evaluation of Some Novel 2-Substituted-3-allyl-4(3H) quinazolinone Derivatives as Anticonvulsant Agents. Med Chem (Los Angeles) 6 : 593-603. doi:10.4172/2161-0444.1000404

Copyright: (c) 2016 Abulkhair HS, et al. This is an open-access article distributed under the terms of the Creative Commons Attribution License, which permits unrestricted use, distribution, and reproduction in any medium, provided the original author and source are credited. 
discs). Either Gemini 300MHz or (Varian USA) were used to record the ${ }^{1} \mathrm{HNMR}$ spectra using TMS as an internal standard. Shimadzu GCMSQP 1000 EX mass spectrometer was used to record mass spectra. Elemental analysis was determined at the Regional Center for Mycology and Biotechnology, Al-Azhar University, Egypt. Starting materials were purchased from Aldrich Chemical Company and used without further purification. 3-Allyl-2,3-dihydro-2-thioxoquinazolin-4(1H)-one (3) was prepared according to reported procedure. Alkyl chloroacetates, chloroacetone, a-bromoacetophenones, hydrazinehydrate and aldehydes were purchased from Aldrich Chemical Company and used without further purification. Anticonvulsant evaluation was carried out at the Department of Toxicology and Pharmacology, Faculty of Pharmacy, Al-Azhar University. Scheme 1 was adopted for synthesis of the new 2-substituted-3-allyl-4(3H)-quinazolinone derivatives. Progress of the reactions was monitored by TLC sheets and was visualized using UV lamp and n-hexane: ethyl acetate 9: 1 as mobile phase.

\section{Preparation of 3-Allyl-2-((2-substituted-2-oxoethyl) thio) quinazolin- $4(3 H)$-one $\left(4_{\mathrm{a}-\mathrm{e}}\right)$}

General procedure: A mixture of equimolar amounts of 3-allyl1,2-dihydro-2-thioxo-4(3H)-quinazolinone (3) (2.18 gm, $0.01 \mathrm{~mol})$, $\alpha$-chloroacetone and/or the appropriate $\alpha$-bromoacetophenone $(0.01$ $\mathrm{mol})$ in DMF $(10 \mathrm{ml})$ containing $\mathrm{K}_{2} \mathrm{CO}_{3}(2 \mathrm{gm})$ was heated on a waterbath with continuous stirring for $6 \mathrm{hrs}$. The reaction mixture was cooled and poured into crushed ice in water $(20 \mathrm{ml})$. The solid was collected, washed with excess water and crystallized from acetic acid to produce the corresponding ketone derivatives $4_{\text {a-e }}$.

4: 3-Allyl-2-((2-methyl-2-oxoethyl) thio) quinazolin-4(3H)-one: Yield (2.05 g, 75\%), m.p. $65-67^{\circ} \mathrm{C} . \mathrm{C}_{14} \mathrm{H}_{14} \mathrm{~N}_{2} \mathrm{O}_{2} \mathrm{~S}$ (274), Analysis \% Calcd. (Found.), C: 61.29 (61.35), H: 5.14 (5.11), N: 10.21 (10.42). IR (KBr, $\left.\mathrm{cm}^{-1}\right): 3085$ (C-H aromatic), 2923 (C-H aliphatic), 1723 (C=O ketone), 1677 (C=O quinazoline). MS (m/z, abund. \%): $274\left(\mathrm{M}^{+}, 11.99 \%\right), 259$ (17.59\%), 231 (56.73\%), 185 (96.37\%), 162 (100\%). ${ }^{1} \mathrm{HNMR}\left(\mathrm{CDCl}_{3}\right.$, ppm): $2.42\left(\mathrm{~s}, 3 \mathrm{H},-\mathrm{COCH}_{3}\right), 4.04\left(\mathrm{~s}, 2 \mathrm{H}, \mathrm{SCH}_{2}\right), 4.78\left(\mathrm{~d}, 2 \mathrm{H}, \mathrm{NCH}_{2}-\right.$ $\mathrm{CH}=), 5.30\left(\mathrm{~m}, 2 \mathrm{H}, \mathrm{CH}=\mathbf{C H}_{2}\right), 5.96\left(\mathrm{~m}, \mathrm{H}, \mathrm{CH}_{2} \mathbf{C H}=\mathrm{CH}_{2}\right), 7.39(\mathrm{dd}, \mathrm{H}$,

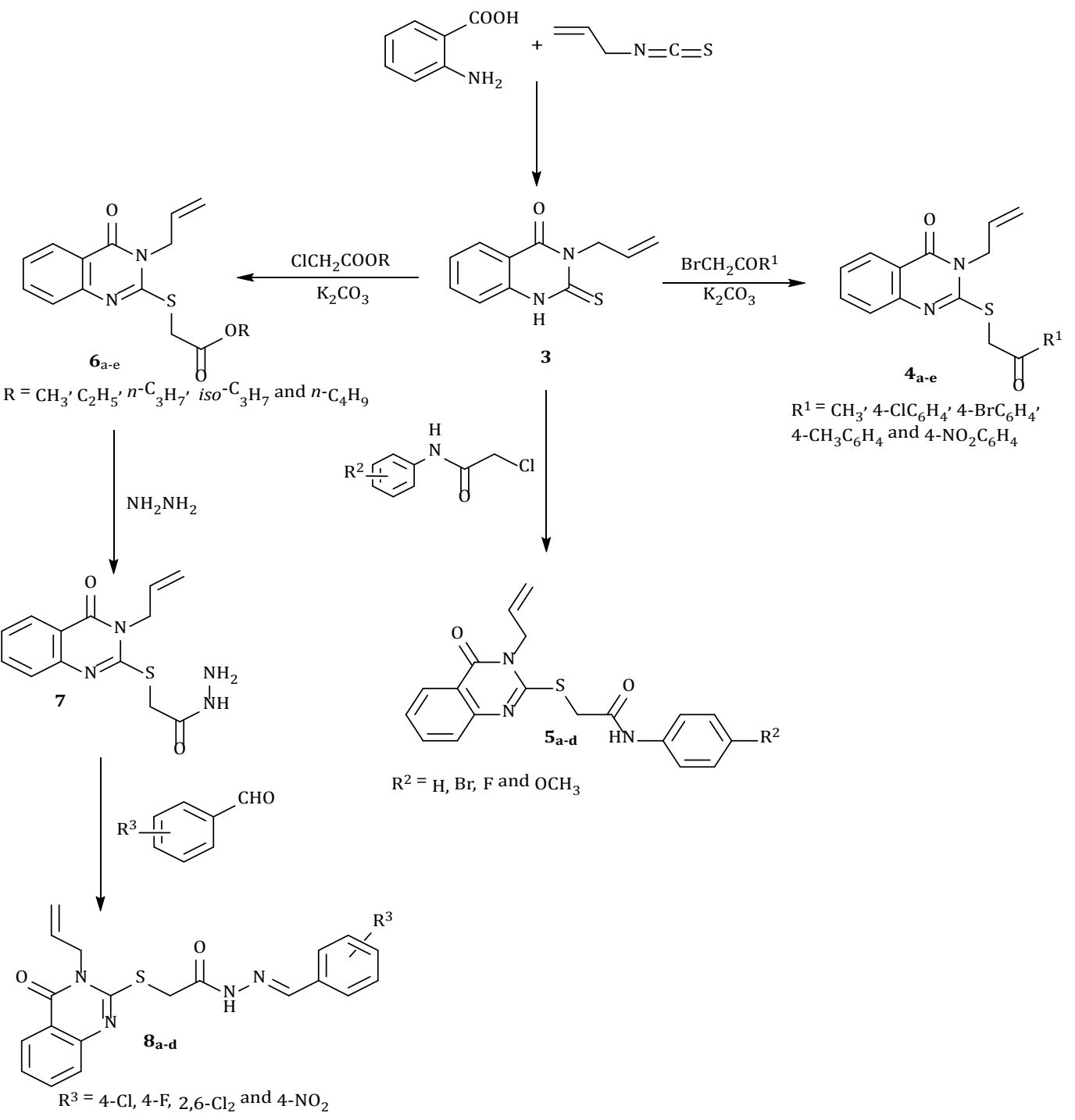

Scheme 1: Synthetic protocol of compounds 3-8. 
Citation: Abulkhair HS, El-Gamal KM, El-Adl K, Fadl MF (2016) Molecular Docking, Synthesis and Biological Evaluation of Some Novel 2-Substituted3-allyl-4(3H)-quinazolinone Derivatives as Anticonvulsant Agents. Med Chem (Los Angeles) 6: 593-603. doi:10.4172/2161-0444.1000404

H-6 quinaz.), 7.47 (d, J = 12.36 Hz, H, H-8 quinaz.), 7.66 (dd, $\mathrm{H}, \mathrm{H}-7$ quinaz.), 8.21 (d, $J=11.91 \mathrm{~Hz}, \mathrm{H}, \mathrm{H}-5$ quinaz.).

$4_{\mathrm{b}}:$ 3-Allyl-2-((2-(4-chlorophenyl)-2-oxoethyl) thio) quinazolin4(3H)-one: Yield (1.85 g, 50\%), m.p. $175-177^{\circ} \mathrm{C} . \mathrm{C}_{19} \mathrm{H}_{15} \mathrm{ClN}_{2} \mathrm{O}_{2} \mathrm{~S}$ (370.5), Analysis \% Calcd. (Found.), C: 61.54 (61.50), H: 4.08 (4.10), N: 7.55 (7.56). IR ( $\left.\mathrm{KBr}, \mathrm{cm}^{-1}\right): 3081$ (C-H aromatic), 2921 (C-H aliphatic), $1682(2 \mathrm{C}=\mathrm{O})$. MS (m/z, abund. \%): $371\left(\mathrm{M}^{+}+1,1.91 \%\right), 370\left(\mathrm{M}^{+}\right.$, 4.34\%), 372 (1.50\%), 259 (12.25\%), 231 (53.11\%), 139 (100\%). ${ }^{1} \mathrm{HNMR}$ $\left(\mathrm{CDCl}_{3}, \mathrm{ppm}\right): 4.60\left(\mathrm{~s}, 2 \mathrm{H}, \mathrm{SCH}_{2}\right), 4.80\left(\mathrm{~d}, 2 \mathrm{H}, \mathrm{NCH}_{2}-\mathrm{CH}=\right), 5.30(\mathrm{~m}$, $\left.2 \mathrm{H}, \mathrm{CH}=\mathbf{C H}_{2}\right), 5.96\left(\mathrm{~m}, \mathrm{H}, \mathrm{CH}_{2} \mathbf{C H}=\mathrm{CH}_{2}\right), 7.10-8.20(\mathrm{~m}, 8 \mathrm{H}$, aromatic protons).

4: 3-Allyl-2-((2-(4-bromophenyl)-2-oxoethyl)thio)quinazolin4(3H)-one: Yield (1.86 g, 45\%), m.p. $142-144^{\circ} \mathrm{C} . \mathrm{C}_{19} \mathrm{H}_{15} \mathrm{BrN}_{2} \mathrm{O}_{2} \mathrm{~S}$ (415), Analysis \% Calcd. (Found.), C: 54.95(54.89), H: 3.64(3.62), N: 6.75(6.79). IR ( $\left.\mathrm{KBr}, \mathrm{cm}^{-1}\right): 3064$ (C-H aromatic), 2916 (C-H aliphatic), $1680(2 \mathrm{C}=\mathrm{O})$. MS (m/z, abund. \%): $416\left(\mathrm{M}^{+}+1,1.59 \%\right), 415\left(\mathrm{M}^{+}\right.$, 2.37\%), 231 (27.59\%), 217 (41.26\%), 185 (100\%), 162 (8.12\%). ${ }^{1} \mathrm{HNMR}$ $\left(\mathrm{CDCl}_{3}, \mathrm{ppm}\right): 4.64\left(\mathrm{~s}, 2 \mathrm{H}, \mathrm{SCH}_{2}\right), 4.84\left(\mathrm{~d}, 2 \mathrm{H}, \mathrm{NCH}_{2}-\mathrm{CH}=\right), 5.33(\mathrm{~m}$, $\left.2 \mathrm{H}, \mathrm{CH}=\mathbf{C H}_{2}\right), 5.98\left(\mathrm{~m}, \mathrm{H}, \mathrm{CH}_{2} \mathbf{C H}=\mathrm{CH}_{2}\right), 7.09-8.19(\mathrm{~m}, 8 \mathrm{H}$, aromatic protons).

4 : 3-Allyl-2-((2-(4-methylphenyl)-2-oxoethyl)thio)quinazolin4(3H)-one: Yield (1.75 g, 50\%), m.p. $180-182^{\circ} \mathrm{C}$. $\mathrm{C}_{20} \mathrm{H}_{18} \mathrm{~N}_{2} \mathrm{O}_{2} \mathrm{~S}(350)$, Analysis \% Calcd. (Found.), C: 68.55 (68.53), H: 5.18 (5.15), N: 7.99 (7.93). IR ( $\left.\mathrm{KBr}, \mathrm{cm}^{-1}\right): 3060$ (C-H aromatic), 2923 (C-H aliphatic), $1682(2 \mathrm{C}=\mathrm{O}) .{ }^{1} \mathrm{HNMR}\left(\mathrm{CDCl}_{3}, \mathrm{ppm}\right): 2.47\left(\mathrm{~s}, 3 \mathrm{H}, \mathrm{CH}_{3}\right), 4.72(\mathrm{~s}, 2 \mathrm{H}$, $\left.\mathrm{SCH}_{2}\right), 4.86\left(\mathrm{~d}, 2 \mathrm{H}, \mathrm{NCH}_{2}-\mathrm{CH}=\right), 5.32\left(\mathrm{~m}, 2 \mathrm{H}, \mathrm{CH}=\mathbf{C H}_{2}\right), 5.98(\mathrm{~m}, \mathrm{H}$, $\left.\mathrm{CH}_{2} \mathbf{C H}=\mathrm{CH}_{2}\right), 7.20-8.21$ (m, $8 \mathrm{H}$, aromatic protons).

4: 3-Allyl-2-((2-(4-nitrophenyl)-2-oxoethyl)thio)quinazolin4(3H)-one: Yield (2.09 g, 55\%), m.p. $145-147^{\circ} \mathrm{C} . \mathrm{C}_{19} \mathrm{H}_{15} \mathrm{~N}_{3} \mathrm{O}_{4} \mathrm{~S}(381)$, Analysis \% Calcd. (Found.), C: 59.83 (59.87), H: 3.96 (3.80), N: 11.02 (11.09). IR ( $\left.\mathrm{KBr}, \mathrm{cm}^{-1}\right): 3078$ (C-H aromatic), 2937 (C-H aliphatic), $1676(2 \mathrm{C}=\mathrm{O})$. MS (m/z, abund. \%): $381\left(\mathrm{M}^{+}, 10.50 \%\right), 259(10.40 \%)$, 231 (48.40\%), 185 (100\%), 162 (8.12\%). ${ }^{1} \mathrm{HNMR}\left(\mathrm{CDCl}_{3}, \mathrm{ppm}\right): 4.74$ (s, $\left.2 \mathrm{H}, \mathrm{SCH}_{2}\right), 4.82\left(\mathrm{~d}, 2 \mathrm{H}, \mathrm{NCH}_{2}-\mathrm{CH}=\right), 5.31\left(\mathrm{~m}, 2 \mathrm{H}, \mathrm{CH}=\mathbf{C H}_{2}\right), 5.94(\mathrm{~m}$, $\left.\mathrm{H}, \mathrm{CH}_{2} \mathbf{C H}=\mathrm{CH}_{2}\right), 7.42-8.24(\mathrm{~m}, 8 \mathrm{H}$, aromatic protons).

Preparation of 2-((3-Allyl-4-oxo-3,4-dihydroquinazolin-2yl)thio)- $\mathrm{N}$-(4-substitutedphenyl)acetamide $\left(5_{\mathrm{a}-\mathrm{d}}\right)$

General procedure: A mixture of equimolar amounts of 3-allyl1,2-dihydro-2-thioxo-4(3H)-quinazolinone (3) $(2.18 \mathrm{gm}, 0.01 \mathrm{~mol})$ and the appropriate $\alpha$-chloroacetanilide derivatives $(0.01 \mathrm{~mol})$ in DMF $(10 \mathrm{ml})$ containing $\mathrm{K}_{2} \mathrm{CO}_{3}(2 \mathrm{gm})$ was heated on a water-bath for $6 \mathrm{hrs}$ with continuous stirring. The reaction mixture was then cooled poured into cold water. The obtained solid was collected, dried and crystallized from ethanol to get the corresponding amide derivatives $\mathbf{5}_{\mathrm{a}-\mathrm{d}}$.

5 : 2-((3-Allyl-4-oxo-3,4-dihydroquinazolin-2-yl)thio)-Nphenylacetamide: Yield $(2.03$ g, $58 \%)$, m.p. $170-172^{\circ} \mathrm{C}$. $\mathrm{C}_{19} \mathrm{H}_{17} \mathrm{~N}_{3} \mathrm{O}_{2} \mathrm{~S}$ (351), Analysis \% Calcd. (Found.), C: 64.94 (64.83), H: 4.88 (4.80), N: 11.96 (12.02). IR ( $\left.\mathrm{KBr}, \mathrm{cm}^{-1}\right): 3328(\mathrm{NH}), 3071$ (C-H aromatic), 2934 (C-H aliphatic), $1670(2 \mathrm{C}=\mathrm{O})$. MS ( $\mathrm{m} / \mathrm{z}$, abund. \%): $352\left(\mathrm{M}^{+}+1,8.30 \%\right)$, 217 (15.10\%), 185 (19.70\%), 259 (100\%). ${ }^{1} \mathrm{HNMR}\left(\mathrm{CDHCl}_{3}, \mathrm{ppm}\right): 4.02$ $\left(\mathrm{s}, 2 \mathrm{H}, \mathrm{SCH}_{2}\right), 4.83\left(\mathrm{~d}, 2 \mathrm{H}, \mathrm{NCH}_{2}-\mathrm{CH}=\right), 5.34\left(\mathrm{~m}, 2 \mathrm{H}, \mathrm{CH}=\mathbf{C H}_{2}, 5.98\right.$ $\left(\mathrm{m}, \mathrm{H}, \mathrm{CH}_{2} \mathrm{CH}=\mathrm{CH}_{2}\right), 7.05-8.31(\mathrm{~m}, 9 \mathrm{H}$, aromatic protons $), 9.79(\mathrm{~s}, 1 \mathrm{H}$, $\mathrm{NH})$.

$5_{b}: 2-((3-A l l y l-4-o x o-3,4-d i h y d r o q u i n a z o l i n-2-y l)$ thio $)-\mathrm{N}$ (4-bromophenyl)acetamide: Yield $(3.01 \mathrm{~g}, 70 \%)$, m.p. $137-139^{\circ} \mathrm{C}$. $\mathrm{C}_{19} \mathrm{H}_{16} \mathrm{BrN}_{3} \mathrm{O}_{2} \mathrm{~S}$ (430), Analysis \% Calcd. (Found.), C: 53.03 (52.92), H: 3.75 (3.76), N: 9.76 (9.72). IR (KBr, $\left.\mathrm{cm}^{-1}\right): 3244(\mathrm{NH}), 3081(\mathrm{C}-\mathrm{H}$ aromatic), 2933 (C-H aliphatic), 1688 (2 C=O). MS (m/z, abund. \%): $432\left(\mathrm{M}^{+}+2,4.80 \%\right), 430\left(\mathrm{M}^{+}, 4.60 \%\right), 432(4.60 \%), 259(100 \%) .{ }^{1} \mathrm{HNMR}$ $\left(\mathrm{CDCl}_{3}, \mathrm{ppm}\right): 4.02\left(\mathrm{~s}, 2 \mathrm{H}, \mathrm{SCH}_{2}\right), 4.82\left(\mathrm{~d}, 2 \mathrm{H}, \mathrm{NCH}_{2}-\mathrm{CH}=\right), 5.35(\mathrm{~m}$, $2 \mathrm{H}, \mathrm{CH}=\mathbf{C H}_{2)}, 5.95\left(\mathrm{~m}, \mathrm{H}, \mathrm{CH}_{2} \mathbf{C H}=\mathrm{CH}_{2}\right), 6.94-8.30(\mathrm{~m}, 8 \mathrm{H}$, aromatic protons), 9.81 (s, 1H, NH).

5 : 2-((3-Allyl-4-oxo-3,4-dihydroquinazolin-2-yl)thio)- $\mathrm{N}$ (4-fluorophenyl)acetamide: Yield (2.58 g, 70\%), m.p. $163-165^{\circ} \mathrm{C}$. $\mathrm{C}_{19} \mathrm{H}_{16} \mathrm{FN}_{3} \mathrm{O}_{2} \mathrm{~S}$ (369), Analysis \% Calcd. (Found.), C: 61.78 (61.75), H: 4.37 (4.39), N: 11.38 (11.35). IR (KBr, cm $\left.{ }^{-1}\right): 3330(\mathrm{NH}), 3068(\mathrm{C}-\mathrm{H}$ aromatic), 2918 (C-H aliphatic), $1671(2 \mathrm{C}=\mathrm{O})$. MS ( $\mathrm{m} / \mathrm{z}$, abund. \%): $370\left(\mathrm{M}^{+}+1,6.00 \%\right), 259$ (100\%), 217 (10.00\%), 185 (15.70\%). ${ }^{1} \mathrm{HNMR}$ $\left(\mathrm{CDCl}_{3}, \mathrm{ppm}\right): 4.03\left(\mathrm{~s}, 2 \mathrm{H}, \mathrm{SCH}_{2}\right), 4.83\left(\mathrm{~d}, 2 \mathrm{H}, \mathrm{NCH}_{2}-\mathrm{CH}=\right), 5.36(\mathrm{~m}$, $2 \mathrm{H}, \mathrm{CH}=\mathbf{C H}_{2}, 5.93\left(\mathrm{~m}, \mathrm{H}, \mathrm{CH}_{2} \mathbf{C H}=\mathrm{CH}_{2}\right), 6.94-8.30(\mathrm{~m}, 8 \mathrm{H}$, aromatic protons), $9.82(\mathrm{~s}, 1 \mathrm{H}, \mathrm{NH})$.

5: 2-((3-Allyl-4-oxo-3,4-dihydroquinazolin-2-yl)thio)-N-(4methoxyphenyl)acetamide: Yield $(2.28 \mathrm{~g}, 60 \%)$, m.p. $152-154^{\circ} \mathrm{C}$ $\mathrm{C}_{20} \mathrm{H}_{10} \mathrm{~N}_{3} \mathrm{O}_{3} \mathrm{~S}$ (381), Analysis \% Calcd. (Found.), C: 62.98 (62.90), H: $5.02(5.15), \mathrm{N}: 11.02(11.04)$. IR $\left(\mathrm{KBr}, \mathrm{cm}^{-1}\right): 3272(\mathrm{NH}), 3072(\mathrm{C}-\mathrm{H}$ aromatic), 2940 (C-H aliphatic), $1692(2 \mathrm{C}=\mathrm{O})$. MS (m/z, abund. \%): 381 (3.60\%), 185 (18.20\%), 259 (100\%). ${ }^{1} \mathrm{HNMR}\left(\mathrm{CDCl}_{3}, \mathrm{ppm}\right): 3.79$ $\left(\mathrm{s}, 3 \mathrm{H}, \mathrm{OCH}_{3}\right), 4.03\left(\mathrm{~s}, 2 \mathrm{H}, \mathrm{SCH}_{2}\right), 4.80\left(\mathrm{~d}, 2 \mathrm{H}, \mathrm{NCH}_{2}-\mathrm{CH}=\right), 5.31(\mathrm{~m}$, $2 \mathrm{H}, \mathrm{CH}=\mathbf{C H}_{2}, 5.96\left(\mathrm{~m}, \mathrm{H}, \mathrm{CH}_{2} \mathbf{C H}=\mathrm{CH}_{2}\right), 6.92-8.31(\mathrm{~m}, 8 \mathrm{H}$, aromatic protons), $9.82(\mathrm{~s}, 1 \mathrm{H}, \mathrm{NH})$.

Preparation of Alkyl 2-((3-allyl-4-oxo-3,4-dihydroquinazolin-2-yl) thio)acetate $\left(6_{\mathrm{a}-\mathrm{e}}\right)$

General procedure: A mixture of $3(2.18 \mathrm{gm}, 0.01 \mathrm{~mol})$ and the appropriate alkyl $\alpha$-chloroacetate $(0.01 \mathrm{~mol})$ in DMF $(20 \mathrm{ml})$ in the presence of anhydrous $\mathrm{K}_{2} \mathrm{CO}_{3}(2 \mathrm{gm})$ was heated on water-bath with continuous stirring for $6 \mathrm{~h}$ and the solid so obtained was filtered, dried and crystallized from ethanol to afford the corresponding ester derivatives $\mathbf{6}_{\mathrm{a}-\mathrm{e}}$.

6: Methyl 2-((3-allyl-4-oxo-3,4-dihydroquinazolin-2-yl)thio) acetate: Yield (1.30 g, 45\%), m.p. $65-67^{\circ} \mathrm{C} . \mathrm{C}_{14} \mathrm{H}_{14} \mathrm{~N}_{2} \mathrm{O}_{3} \mathrm{~S}(290)$, Analysis \% Calcd. (Found.): C: 57.92 (57.99), H: 4.86 (4.80), N: 9.65 (9.80). IR $\left(\mathrm{KBr}, \mathrm{cm}^{-1}\right): 3068$ (C-H aromatic), 2926 (C-H aliphatic), 1745 (C=O ester), 1687 ( $\mathrm{C}=\mathrm{O}$ quinazoline). $\mathrm{MS}$ ( $\mathrm{m} / \mathrm{z}$, abund. \%): $290\left(\mathrm{M}^{+}, 1.94 \%\right)$, 217 (9.38\%), 59 (100\%). ${ }^{1} \mathrm{HNMR}\left(\mathrm{CDCl}_{3}, \mathrm{ppm}\right): 3.79$ (s, 3H, $\left.\mathrm{OCH}_{3}\right)$, $4.04\left(\mathrm{~s}, 2 \mathrm{H}, \mathrm{SCH}_{2}\right), 4.78\left(\mathrm{~d}, J=9.6 \mathrm{~Hz}, 2 \mathrm{H}, \mathrm{NCH}_{2}-\mathrm{CH}=\right), 5.31(\mathrm{~m}, J=$ $9.6 \mathrm{~Hz}, 2 \mathrm{H}, \mathrm{CH}=\mathrm{CH}_{2}, 5.95\left(\mathrm{~m}, \mathrm{H}, \mathrm{CH}_{2} \mathbf{C H}=\mathrm{CH}_{2}\right), 7.39$ (dd, H, H-6 quinaz.), 7.51 (d, H, H-8 quinaz.), 7.69 (dd, $\mathrm{H}, \mathrm{H}-7$ quinaz.), 8.20 (d, $J$ $=9.6 \mathrm{~Hz}, \mathrm{H}, \mathrm{H}-5$ quinaz.).

6: Ethyl 2-((3-allyl-4-oxo-3,4-dihydroquinazolin-2-yl)thio) acetate: Yield $(3.38$ g, $70 \%)$, m.p. $70-72^{\circ} \mathrm{C}$ as. $\mathrm{C}_{15} \mathrm{H}_{16} \mathrm{~N}_{2} \mathrm{O}_{3} \mathrm{~S}(304)$, Analysis \% Calcd. (Found.): C: 59.19 (59.22), H: 5.30 (5.30), N: 9.20 (9.21). IR ( $\left.\mathrm{KBr}, \mathrm{cm}^{-1}\right): 3067$ (C-H aromatic), 2935 (C-H aliphatic), 1747 (C=O ester), 1683 (C=O quinazoline). MS (m/z, abund. \%): $304\left(\mathrm{M}^{+}\right.$, 12.98\%), 217 (60.23\%), 185 (100\%). ${ }^{1} \mathrm{HNMR}\left(\mathrm{CDCl}_{3}, \mathrm{ppm}\right): 1.28$ (t, 3H, $\left.\mathrm{CH}_{3}\right), 4.00\left(\mathrm{~s}, 2 \mathrm{H}, \mathrm{SCH}_{2}\right), 4.28\left(\mathrm{q}, 2 \mathrm{H}, \mathrm{OCH}_{2}-\mathrm{CH}_{3}, 4.78\right.$ (d, $2 \mathrm{H}, \mathrm{NCH}_{2}-$ $\mathrm{CH}=), 5.30\left(\mathrm{~m}, 2 \mathrm{H}, \mathrm{CH}=\mathbf{C H}_{2}, 5.93\left(\mathrm{~m}, \mathrm{H}, \mathrm{CH}_{2} \mathbf{C H}=\mathrm{CH}_{2}\right), 7.36(\mathrm{dd}, \mathrm{H}\right.$, $\mathrm{H}-6$ quinaz.), 7.48 (d, $J=11.88 \mathrm{~Hz}, \mathrm{H}, \mathrm{H}-8$ quinaz.), 7.66 (dd, $\mathrm{H}, \mathrm{H}-7$ quinaz.), 8.20 (d, $J=11.97 \mathrm{~Hz}, \mathrm{H}, \mathrm{H}-5$ quinaz.).

6: Propyl 2-((3-allyl-4-oxo-3,4-dihydroquinazolin-2-yl)thio) acetate: Yield $(1.90$ g, $60 \%)$, m.p. $68-70^{\circ} \mathrm{C} . \mathrm{C}_{16} \mathrm{H}_{18} \mathrm{~N}_{2} \mathrm{O}_{3} \mathrm{~S}(318)$, Analysis \% Calcd. (Found.), C: 60.36 (60.32), H: 5.70 (5.75), N: 8.80 (8.76). IR $\left(\mathrm{KBr}, \mathrm{cm}^{-1}\right): 3062$ (C-H aromatic), 2945 (C-H aliphatic), $1741(\mathrm{C}=\mathrm{O}$ ester), 1682 ( $\mathrm{C}=\mathrm{O}$ quinazoline). $\mathrm{MS}$ ( $\mathrm{m} / \mathrm{z}$, abund. \%): $318\left(\mathrm{M}^{+}, 9.09 \%\right)$, 217 (58.70\%), 185 (100\%). ${ }^{1} \mathrm{HNMR}\left(\mathrm{CDCl}_{3}, \mathrm{ppm}\right): 0.8(\mathrm{t}, J=9.6 \mathrm{~Hz}, 3 \mathrm{H}$, $\left.\mathrm{CH}_{2}-\mathbf{C H}_{3}\right), 1.70\left(\mathrm{~m}, J=9.6 \mathrm{~Hz}, 2 \mathrm{H}, \mathbf{C H}_{2}-\mathrm{CH}_{3}\right), 4.04\left(\mathrm{~s}, 2 \mathrm{H}, \mathrm{SCH}_{2}\right), 4.14$ 
Citation: Abulkhair HS, El-Gamal KM, El-Adl K, Fadl MF (2016) Molecular Docking, Synthesis and Biological Evaluation of Some Novel 2-Substituted3-allyl-4(3H)-quinazolinone Derivatives as Anticonvulsant Agents. Med Chem (Los Angeles) 6: 593-603. doi:10.4172/2161-0444.1000404

$\left(\mathrm{t}, J=9.6 \mathrm{~Hz}, 2 \mathrm{H}, \mathrm{OCH}_{2}-\mathrm{CH}_{2}, 4.80\left(\mathrm{~d}, 2 \mathrm{H}, \mathrm{NCH}_{2}-\mathrm{CH}=\right), 5.30(\mathrm{~m}, 2 \mathrm{H}\right.$, $\mathrm{CH}=\mathbf{C H}_{2}, 5.94\left(\mathrm{~m}, \mathrm{H}, \mathrm{CH}_{2} \mathbf{C H}=\mathrm{CH}_{2}\right), 7.41$ (dd, $\mathrm{H}, \mathrm{H}-6$ quinaz. $), 7.46$ (d, $J=11.91 \mathrm{~Hz}, \mathrm{H}, \mathrm{H}-8$ quinaz.), 7.68 (dd, $\mathrm{H}, \mathrm{H}-7$ quinaz.), 8.22 (d, $J=$ $11.91 \mathrm{~Hz}, \mathrm{H}, \mathrm{H}-5$ quinaz.).

6. Isopropyl 2-((3-allyl-4-oxo-3,4-dihydroquinazolin-2-yl)thio) acetate: Yield (2.06 g, 65\%), m.p. $65-67^{\circ} \mathrm{C} . \mathrm{C}_{16} \mathrm{H}_{18} \mathrm{~N}_{2} \mathrm{O}_{3} \mathrm{~S}(318)$, Analysis \% Calcd. (Found.), C: 60.36 (60.25), H: 5.70 (5.87), N: 8.80 (8.70). IR $\left(\mathrm{KBr}, \mathrm{cm}^{-1}\right): 3061$ (C-H aromatic), 2984 (C-H aliphatic), $1735(\mathrm{C}=\mathrm{O}$ ester), $1684(\mathrm{C}=\mathrm{O}$ quinazoline $)$. MS ( $\mathrm{m} / \mathrm{z}$, abund. \%): $318\left(\mathrm{M}^{+}, 10.27 \%\right)$, 217 (72.70\%), 185 (100\%). ${ }^{1} \mathrm{HNMR}\left(\mathrm{CDCl}_{3}, \mathrm{ppm}\right): 1.25,1.28(2 \mathrm{~s}, 6 \mathrm{H}$, $\left.\mathrm{CH}\left(\mathrm{CH}_{3}\right)_{2}\right), 3.99\left(\mathrm{~s}, 2 \mathrm{H}, \mathrm{SCH}_{2}\right), 4.80\left(\mathrm{~d}, 2 \mathrm{H}, \mathrm{NCH}_{2}-\mathrm{CH}=\right), 5.08(\mathrm{~m}, 2 \mathrm{H}$, $\mathrm{OCH}), 5.31\left(\mathrm{~m}, 2 \mathrm{H}, \mathbf{C H}=\mathbf{C H}_{2}, 5.97\left(\mathrm{~m}, \mathrm{H}, \mathrm{CH}_{2} \mathbf{C H}=\mathrm{CH}_{2}\right), 7.37(\mathrm{dd}, \mathrm{H}\right.$, H-6 quinaz.), 7.46 (d, $J=11.55 \mathrm{~Hz}, \mathrm{H}, \mathrm{H}-8$ quinaz.), 7.67 (dd, $\mathrm{H}, \mathrm{H}-7$ quinaz.), 8.22 (d, $J=12.06 \mathrm{~Hz}, \mathrm{H}, \mathrm{H}-5$ quinaz.).

6: Butyl 2-((3-allyl-4-oxo-3,4-dihydroquinazolin-2-yl)thio) acetate: Yield (2.32g, $70 \%)$, m.p. $65-67^{\circ} \mathrm{C} . \mathrm{C}_{17} \mathrm{H}_{20} \mathrm{~N}_{2} \mathrm{O}_{3} \mathrm{~S}(332)$, Analysis \% Calcd. (Found.), C: 61.42 (61.35), H: 6.06 (6.10), N: 8.43 (8.42). IR $\left(\mathrm{KBr}, \mathrm{cm}^{-1}\right): 3073$ (C-H aromatic), 2957 (C-H aliphatic), $1740(\mathrm{C}=\mathrm{O}$ ester), 1675 (C=O quinazoline). MS (m/z, abund. \%): $332\left(\mathrm{M}^{+}, 7.00 \%\right)$, 217 (66.62 \%), 185 (100\%). ${ }^{1} \mathrm{HNMR}\left(\mathrm{CDCl}_{3}, \mathrm{ppm}\right): 0.88$ (t, $J=10.98$ $\left.\mathrm{Hz}, 3 \mathrm{H}, \mathrm{CH}_{2}-\mathbf{C H}_{3}\right), 1.37\left(\mathrm{~m}, J=10.98 \mathrm{~Hz}, 2 \mathrm{H}, \mathbf{C H}_{2}-\mathrm{CH}_{3}\right), 1.67(\mathrm{~m}, 2 \mathrm{H}$, $\left.\mathrm{CH}_{2}-\mathrm{CH}_{2}-\mathrm{CH}_{3}\right), 4.03\left(\mathrm{~s}, 2 \mathrm{H}, \mathrm{SCH}_{2}\right), 4.18\left(\mathrm{t}, 2 \mathrm{H}, \mathrm{OCH}_{2}-\mathrm{CH}_{2}, 4.80(\mathrm{~d}\right.$, $\left.2 \mathrm{H}, \mathrm{NCH}_{2}-\mathrm{CH}=\right), 5.31\left(\mathrm{~m}, 2 \mathrm{H}, \mathrm{CH}=\mathbf{C H}_{2}, 5.97\left(\mathrm{~m}, \mathrm{H}, \mathrm{CH}_{2} \mathbf{C H}=\mathrm{CH}_{2}\right)\right.$, 7.38 (dd, H, H-6 quinaz.), 7.46 (d, $J=11.97 \mathrm{~Hz}, \mathrm{H}, \mathrm{H}-8$ quinaz.), 7.68 (dd, $\mathrm{H}, \mathrm{H}-7$ quinaz.), 8.22 (d, $J=11.97 \mathrm{~Hz}, \mathrm{H}, \mathrm{H}-5$ quinaz.).

Preparation of 2-((3-Allyl-4-oxo-3,4-dihydroquinazolin-2yl)thio)acetohydrazide (7)

A mixture of hydrazine hydrate $(0.64 \mathrm{~g}, 0.2 \mathrm{~mol})$ and 3-allyl-2ethoxy-carbonylmethylthio-4 $(3 \mathrm{H})$-quinazolinone $\left(\mathbf{4}_{\mathrm{b}}\right)$ (3.04 $\mathrm{gm}, 0.1$ mole) in ethanol $(10 \mathrm{ml})$ was heated under reflux for about $6 \mathrm{hrs}$. The mixture was then concentrated by evaporation of the solvent whereby the hydrazide derivative 7 crystallized out as fine pale yellow crystals.

Yield (1.88g, 65\%), m.p. $145-147^{\circ} \mathrm{C} \cdot \mathrm{C}_{13} \mathrm{H}_{14} \mathrm{~N}_{4} \mathrm{O}_{2} \mathrm{~S}$ (290), Analysis \% Calcd. (Found.), C: 53.78 (53.64), H: 4.86 (4.90), N: 19.30 (19.32). IR $\left(\mathrm{KBr}, \mathrm{cm}^{-1}: 3279\left(\mathrm{NH}_{2}\right), 3230(\mathrm{NH}), 3037\right.$ (C-H aromatic), 2917 (C-H aliphatic), 1681 ( $\mathrm{C}=\mathrm{O}$ quinazoline $), 1658(\mathrm{C}=\mathrm{O}$ hydrazide $) . \mathrm{MS}(\mathrm{m} / \mathrm{z}$, abund. \%): 290 (M+, 4.75\%), 259 (100\%), 217 (9.17\%), 203 (61.47\%), 185 (10.59\%). ${ }^{1} \mathrm{HNMR}\left(\mathrm{CDCl}_{3}, \mathrm{ppm}\right): 3.97$ (s, 2H, SCH $), 4.37$ (s, 2H, $\mathrm{NH}_{2}, \mathrm{D}_{2} \mathrm{O}$ exchangeable), $4.73\left(\mathrm{~d}, 2 \mathrm{H}, \mathrm{NCH}_{2}-\mathrm{CH}=\right), 5.21(\mathrm{~m}, 2 \mathrm{H}$, $\mathrm{CH}=\mathbf{C H}_{2}, 5.92\left(\mathrm{~m}, \mathrm{H}, \mathrm{CH}_{2} \mathbf{C H}=\mathrm{CH}_{2}\right), 7.43-8.09(\mathrm{~m}, 8 \mathrm{H}$, aromatic protons), 9.36 (s, $1 \mathrm{H}, \mathrm{NH}, \mathrm{D}_{2} \mathrm{O}$ exchangeable).

Preparation of 2-((3-Allyl-4-oxo-3,4-dihydroquinazolin-2yl)thio)- $N$ '-(substitutedbenzylidene)aceto-hydrazide $\left(8_{\mathrm{a}-\mathrm{d}}\right)$.

4.6.1 General procedure: The appropriate aromatic aldehyde $(0.01$ mol) was dissolved in glacial acetic $(15 \mathrm{ml})$ and added to the hydrazide derivative 6 ( $2.9 \mathrm{~g}, 0.01 \mathrm{~mol})$ in acetic acid $(15 \mathrm{ml})$. The reaction mixture was heated under reflux overnight. Solvent was then removed out under reduced pressure, and the solid product was collected, crystallized from ethanol to obtain the corresponding benzylideneacetohydrazide derivative 8

8: $\quad$ 2-((3-Allyl-4-oxo-3,4-dihydroquinazolin-2-yl)thio)-N'-(4chlorobenzylidene)acetohydrazide: Yield (1.85 g, 45\%), m.p. 175$177^{\circ} \mathrm{C}$. $\mathrm{C}_{20} \mathrm{H}_{17} \mathrm{ClN}_{4} \mathrm{O}_{2} \mathrm{~S}$ (412.5), Analysis \% Calcd. (Found.), C: 58.18 (58.14), H: 4.15 (4.13), N: 13.57 (13.41). IR ( $\left.\mathrm{KBr}, \mathrm{cm}^{-1}\right): 3211(\mathrm{NH}), 3045$ (C-H aromatic), 2940 (C-H aliphatic), $1684(2 \mathrm{C}=\mathrm{O})$. MS (m/z, abund. \%): $413\left(\mathrm{M}^{+}+1,2.18 \%\right), 412\left(\mathrm{M}^{+}, 6.44 \%\right), 259$ (33.97\%), $203(100 \%)$, 185 (75.33\%). ${ }^{1} \mathrm{HNMR}\left(\mathrm{CDCl}_{3}, \mathrm{ppm}\right): 4.11$ (s, $\left.2 \mathrm{H}, \mathrm{SCH}_{2}\right), 4.75$ (d, $2 \mathrm{H}$,
$\left.\mathrm{NCH}_{2}-\mathrm{CH}=\right), 5.23\left(\mathrm{~m}, 2 \mathrm{H}, \mathrm{CH}=\mathbf{C H}_{2}, 5.96\left(\mathrm{~m}, \mathrm{H}, \mathrm{CH}_{2} \mathbf{C H}=\mathrm{CH}_{2}\right), 7.30-\right.$ $8.33(\mathrm{~m}, 8 \mathrm{H}$, aromatic protons), $8.42(\mathrm{~s}, \mathrm{H}, \mathrm{N}=\mathrm{CH}), 11.93(\mathrm{~s}, 1 \mathrm{H}, \mathrm{NH})$.

$8_{\mathrm{b}}: \quad$ 2-((3-Allyl-4-oxo-3,4-dihydroquinazolin-2-yl)thio)-N'-(4fluorobenzylidene)acetohydrazide: Yield (1.38 g, 35\%), m.p. 170$172^{\circ} \mathrm{C} . \mathrm{C}_{20} \mathrm{H}_{17} \mathrm{FN}_{4} \mathrm{O}_{2} \mathrm{~S}$ (396), Analysis \% Calcd. (Found.), C: 60.59 (60.62), H: 4.32 (4.35), N: 14.13 (14.08). IR $\left(\mathrm{KBr}, \mathrm{cm}^{-1}\right): 3231(\mathrm{NH})$ 3063 (C-H aromatic), 2927 (C-H aliphatic), 1683 (C=O quinazoline), 1656 (C=O hydrazide). MS (m/z, abund. \%): $396\left(\mathrm{M}^{+}, 1.20 \%\right), 259$ (100\%), 203 (47.49\%), 185 (23.42\%). ${ }^{1} \mathrm{HNMR}\left(\mathrm{CDCl}_{3}, \mathrm{ppm}\right): 4.08$ (s, $\left.2 \mathrm{H}, \mathrm{SCH}_{2}\right), 4.70\left(\mathrm{~d}, 2 \mathrm{H}, \mathrm{NCH}_{2}-\mathrm{CH}=\right), 5.19\left(\mathrm{~m}, 2 \mathrm{H}, \mathrm{CH}=\mathbf{C H}_{2}, 5.99\right.$ $\left(\mathrm{m}, \mathrm{H}, \mathrm{CH}_{2} \mathrm{CH}=\mathrm{CH}_{2}\right), 7.28-8.31(\mathrm{~m}, 8 \mathrm{H}$, aromatic protons $), 8.50(\mathrm{~s}, \mathrm{H}$, $\mathrm{N}=\mathrm{CH}), 11.88(\mathrm{~s}, 1 \mathrm{H}, \mathrm{NH})$.

8: 2-((3-Allyl-4-oxo-3,4-dihydroquinazolin-2-yl)thio)-N'-(2,6dichlorobenzylidene)acetohydrazide: Yield (2.89 g, 65\%), m.p. 220$222^{\circ} \mathrm{C}$. $\mathrm{C}_{20} \mathrm{H}_{16} \mathrm{Cl}_{2} \mathrm{~N}_{4} \mathrm{O}_{2} \mathrm{~S}$ (446), Analysis \% Calcd. (Found.), C: 53.70 (53.66), H: 3.61 (3.55), N: 12.52 (12.43). IR $\left(\mathrm{KBr}, \mathrm{cm}^{-1}\right): 3209(\mathrm{NH})$ 3071 (C-H aromatic), 2926 (C-H aliphatic), $1672(2 \mathrm{C}=\mathrm{O}) . \mathrm{MS}(\mathrm{m} / \mathrm{z}$, abund. \%): $449\left(\mathrm{M}^{+}+3,0.37 \%\right), 448\left(\mathrm{M}^{+}+2,0.42 \%\right), 446\left(\mathrm{M}^{+}, 0.47 \%\right)$, 259 (100\%), 203 (62.16\%), 185 (30.92\%). ${ }^{1} \mathrm{HNMR}\left(\mathrm{CDCl}_{3}, \mathrm{ppm}\right): 4.10$ $\left(\mathrm{s}, 2 \mathrm{H}, \mathrm{SCH}_{2}\right), 4.74\left(\mathrm{~d}, 2 \mathrm{H}, \mathrm{NCH}_{2}-\mathrm{CH}=\right), 5.21\left(\mathrm{~m}, 2 \mathrm{H}, \mathrm{CH}=\mathbf{C H}_{2}, 5.91\right.$ (m, $\left.\mathrm{H}, \mathrm{CH}_{2} \mathrm{CH}=\mathrm{CH}_{2}\right), 7.37-8.08(\mathrm{~m}, 7 \mathrm{H}$, aromatic protons), $8.33(\mathrm{~s}, \mathrm{H}$, $\mathrm{N}=\mathrm{CH}), 11.90(\mathrm{~s}, 1 \mathrm{H}, \mathrm{NH})$.

8: 2-((3-Allyl-4-oxo-3,4-dihydroquinazolin-2-yl)thio)-N'-(4nitrobenzylidene)acetohydrazide: Yield (2.96 g, 70\%), m.p. 173$175^{\circ} \mathrm{C} . \mathrm{C}_{20} \mathrm{H}_{17} \mathrm{~N}_{5} \mathrm{O}_{4} \mathrm{~S}(423)$, Analysis \% Calcd. (Found.), C: 56.73 (56.70), H: 4.05 (4.18), N: 16.54 (16.62). IR (KBr, cm $\left.{ }^{-1}\right): 3179(\mathrm{NH}), 3078(\mathrm{C}-\mathrm{H}$ aromatic), 2933 (C-H aliphatic), $1675(2 \mathrm{C}=\mathrm{O})$. MS (m/z, abund. \%): $423\left(\mathrm{M}^{+}, 1.20 \%\right), 259$ (64.28\%), 203 (100\%). ${ }^{1} \mathrm{HNMR}\left(\mathrm{CDCl}_{3}, \mathrm{ppm}\right)$ : $4.04\left(\mathrm{~s}, 2 \mathrm{H}, \mathrm{SCH}_{2}\right), 4.80\left(\mathrm{~d}, 2 \mathrm{H}, \mathrm{NCH}_{2}-\mathrm{CH}=\right), 5.30\left(\mathrm{~m}, 2 \mathrm{H}, \mathrm{CH}=\mathbf{C H}_{2}\right.$, $5.97\left(\mathrm{~m}, \mathrm{H}, \mathrm{CH}_{2} \mathrm{CH}=\mathrm{CH}_{2}\right), 7.38-8.22(\mathrm{~m}, 8 \mathrm{H}$, aromatic protons $), 8.42$ (s, $\mathrm{H}, \mathrm{N}=\mathrm{CH}), 11.88(\mathrm{~s}, 1 \mathrm{H}, \mathrm{NH})$.

\section{Docking studies}

In the present work, all the target compounds were subjected to docking study to explore their binding mode to GABA-A receptor as a proposed mode of their anticonvulsant activity. All modeling experiments were performed using molsoft (ICM-Pro) program which provides a unique set of tools for the modeling of protein/ligand interactions. It predicts how small flexible molecules such as substrates or drug candidates bind to a protein of known 3D structure represented by grid interaction potentials (http://www.molsoft.com/icm_pro. html). Each experiment used the biological target GABA-A receptor downloaded from the Brookhaven Protein Databank (http://www.rcsb. org/pdb/explore/explore.do?structureId $=4 \mathrm{COF}$ ). In order to qualify the docking results in terms of accuracy of the predicted binding conformations in comparison with the experimental procedure, the reported GABA-A receptor stimulant drug (Methaqualone) was used as a reference ligand. The docking study has been conducted to predict the binding mode and to rationalize the observed biological activity.

\section{Anticonvulsant evaluation}

The animal studies were undertaken with approval from the Ethics Committee (approval \# 23PD/3/12/8R) of Al-Azhar University, Nasr City, Cairo, Egypt. All the trials were carried out according to the respective internationally guidelines. Swiss albino adult male mice, weighing 20-25 g, were used as experimental animals. They were obtained from an animal facility (Animal house, Department of Pharmacology and Toxicology, Faculty of Pharmacy, Al-Azhar University). Mice were housed in stainless steel wire-floored cages 
without any stressful stimuli. Animals were kept under well-ventilated conditions at room temperature $\left(25-30^{\circ} \mathrm{C}\right)$. They were fed on an adequate standard laboratory chow (El-Nasr Co., Abou-Zabal, Egypt) and allowed to acclimatize with free access to food and water for $24 \mathrm{~h}$ period before testing except during the short time they were removed from the cages for testing. Methaqualone and sodium valproate (Sigma-Aldrich Chemical Co, Milwaukee, WI, USA) were used as reference drugs for comparison. The newly synthesized compounds were screened to evaluate their anticonvulsant activity and their neurotoxicity. The anticonvulsant activities of the selected compounds were evaluated by two models, pentylenetetrazole (PTZ), and maximal electroshock (MES) models according to the reported procedures. PTZ (Sigma-Aldrich Chemical Co, Milwaukee, WI, USA) was used to induce convulsions in the experimental animals. The test compounds were dissolved in 10\% DMSO and injected intraperitoneally (i.p.) at a dose of $0.5 \mathrm{mmol} / \mathrm{kg} 30 \mathrm{~min}$ before seizures induction. Methaqualone $(0.8 \mathrm{mmol} / \mathrm{kg})$ and sodium valproate $(1.8 \mathrm{mmol} / \mathrm{kg})$ were used as reference drugs. PTZ (PTZ, Sigma) was dissolved in normal saline in $2 \%$ concentration and was given (i.p.) in a dose of $60 \mathrm{mg} / \mathrm{kg}$ body weight (dose that could induce convulsions in at least $80 \%$ of the animals without death during the following $24 \mathrm{~h}$ ). All drugs were freshly prepared to the desired concentration just before use.

Groups of six mice were administered the graded doses of the test compounds. Control animals received an equal volume of saline (10 $\mathrm{ml} / \mathrm{kg}$ ). After $30 \mathrm{~min}$, the animals were subcutaneously injected with the convulsive dose of PTZ $(60 \mathrm{mg} / \mathrm{kg})$. The criterion of anticonvulsant activity is complete protection against convulsions of any kind. Observations were made at least $60 \mathrm{~min}$ after the administration of PTZ. In the MES test, the electrical stimulus produced from electroconvulsiometer was $50 \mathrm{~mA}, 60 \mathrm{~Hz}$. Current was applied for $0.2 \mathrm{~s}$ via auricular electrodes. Protection against the spread of MESinduced seizures was identified by the abolition of the hind leg and tonic maximal extension component of the seizure.

The highest potent compounds $\mathbf{4}_{\mathfrak{c}}, \mathbf{4}_{\mathrm{b}}$ and $\mathbf{4}_{\mathrm{d}}$ were further evaluated at different doses in PTZ model to determine their protective and therapeutic indexes. Neurotoxicity which was indicated by median toxic dose producing minimal neurological toxicity in $50 \%$ of mice $\left(\mathrm{TD}_{50}\right)$ was calculated (in $\mathrm{mg} / \mathrm{kg}$ ) and used for comparison among compounds under test as shown in Table 3. Neurological toxicity (NT) was determined by rotarod test in mice using the method reported by Dunham and Miya. In brief, a group of animals (mice) were trained to balance on a rotating rod $(3 \mathrm{~cm}$ diameter and $6 \mathrm{rpm}$ speed) and they were allowed three attempts to remain on the rotating rod for $20 \mathrm{~s}$. The trained animals were treated with the tested compounds at various dose levels by i.p. administration. The tested compounds were considered to be neurotoxic at a particular dose level if the trained animal showed lack of Rolling Roller Performance. The trained animals were tested in this manner at $30 \mathrm{~min}$ and $4 \mathrm{~h}$ after the drug administration and then the neurotoxic effect was recorded in terms of median toxic dose $\left(\mathrm{TD}_{50}\right)$. The protective index was determined from the equation $\left(\mathrm{PI}=\mathrm{TD}_{50} / \mathrm{ED}_{50}\right)$. The median lethal dose $\left(\mathrm{LD}_{50}\right)$, the dose that causes 50\% mortality in mice was calculated (in $\mathrm{mg} / \mathrm{kg}$ ) and used for comparison among compounds under test as shown in Table 3. Therapeutic index was determined from the equation $\left(\mathrm{TI}=\mathrm{LD}_{50} / \mathrm{ED}_{50}\right)$.

\section{Results and Discussion}

\section{Rationale and structure-based design}

Two reported facts were analyzed before the chemical synthesis of our target compounds. First fact was: modifications at second and third positions of methaqualone have led to the generation of many CNS active agents such as afloqualone, etaqualone, mebroqualone, mecloqualone and diproqualone (Figure 1). Mecloqualone was found to possess a significant anticonvulsant action 1.5 times more potent than phenytoin against MES-induced convulsions and ten times more potent than troxidone against PTZ-induced seizures [13]. Second fact explained that: replacement of the phenyl group at second position of quinazolin- $4(3 \mathrm{H})$-one by ethyl one and replacement of the methyl group at position 2 by substituted thiazolidine distal moiety linked at 2-position through $\mathrm{SCH}_{2} \mathrm{CONHN}$ and/or $\mathrm{SCH}_{2} \mathrm{CONH}$ linkers yielded good anticonvulsant agents as in compounds (A and B) [16]. Figure 1 represents the structural similarities and pharmacophoric features of some reported anticonvulsants quinazolinones and our designed compounds. Based on the previously mentioned facts, it appeared to us that considerable promise for discovering new anticonvulsants might be found through the synthesis of structural analogs of these compounds.

Figure 1 shows that structure of the title final compounds $\left(4_{\mathrm{a}-\mathrm{e}}^{-}\right.$ $8_{\mathrm{a-d}}$ ) fulfilled all the pharmacophoric structural requirements. These requirements include: the presence of quinazolin-4(3H)-one moiety as hydrophobic portion, $\mathrm{N}$ as electron donor system, the presence of the carbonyl group as hydrogen bonding site, the allyl group at 3-position as hydrophobic domain and the presence of the distal moiety linked at 2-position through $\mathrm{SCH}_{2} \mathrm{CO}, \mathrm{SCH}_{2} \mathrm{COO}, \mathrm{SCH}_{2} \mathrm{CONH}$ and/or $\mathrm{SCH}_{2} \mathrm{CONHN}$ linkers as another hydrophobic domain responsible for controlling the pharmacokinetic properties of the antiepileptic activity.

Our target compounds are designed as hybrid molecules formed of quinazolinone ring system to maintain basic features of the lead structure methaqualone with replacement of the phenyl group at position 3 of synthesized compounds by allyl one and replacement of the methyl group at position 2 by different moieties as hydrophobic portions. These moieties were joined at 2-position through different linkers having different electronic environments to study the SAR of these compounds and the effect of each substituent on their anticonvulsant activity hoping to obtain more potent anticonvulsant agents.

\section{Molecular docking study}

The obtained results indicated that all studied ligands have similar position and orientation inside the putative binding site of GABA-A receptor (PDB code 4COF), as a proposed mode of their anticonvulsant activity, which revealed a large space bounded by a membrane-binding domain which serves as an entry channel for substrate to the active site (Figure 2). In addition, the affinity of any small molecule can be considered as a unique tool in the field of drug design. There is a relationship between the affinity of organic molecules and the free energy of binding. This relationship can contribute in prediction and interpretation of the activity of the organic compounds toward the specific target protein $[17,18]$ The obtained results of the free energy of binding $(\Delta \mathrm{G})$ explained that most of these compounds had good binding affinity toward the receptor and the computed values reflected the overall trend (Table 1).

The proposed binding mode of methaqualone revealed an affinity value of $-50.69 \mathrm{kcal} / \mathrm{mol}$ and formed $2 \mathrm{H}$-bonds with Lysine215 (1.52 $\AA$ ) and Isoleucine $218(1.81 \AA)$. These are key amino acids acting as a gate for ligand entrance to the GABA-A (Figure 3). The quinazolinone moiety occupied the hydrophobic pocket formed by Serine187, Lysine215, Asparagine217, Isoleucine218 and Glycine219. The phenyl ring at position-3 was oriented in the hydrophobic cleft formed by Arginine142, Leucine145, Aspartate146, Glutamate147, Lysine215, 


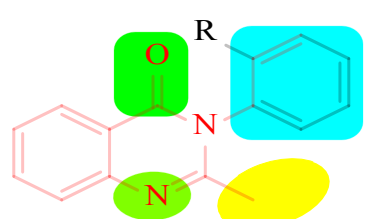

Methaqualone $\mathrm{R}=\mathrm{CH}_{3}$

Etaqualone $\quad \mathrm{R}=\mathrm{C}_{2} \mathrm{H}_{5}$

Mecloqualone $\mathrm{R}=\mathrm{Cl}$

Mebroqualone $\mathrm{R}=\mathrm{Br}$

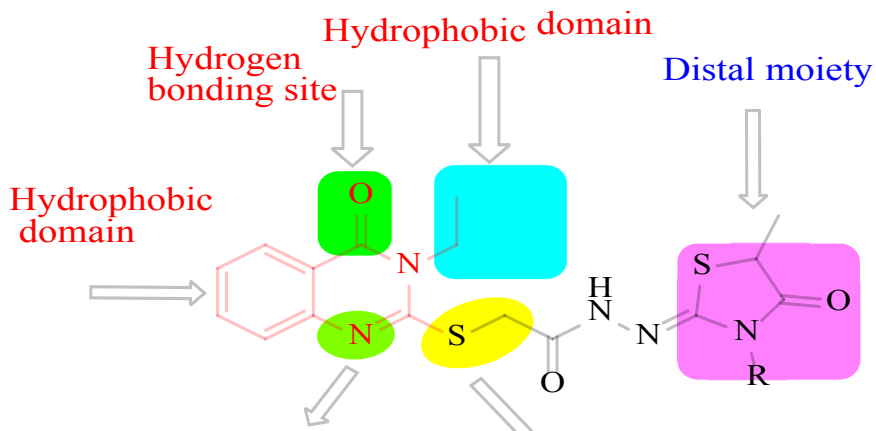

Electron donor moity

Hydrophobic domain

(A)

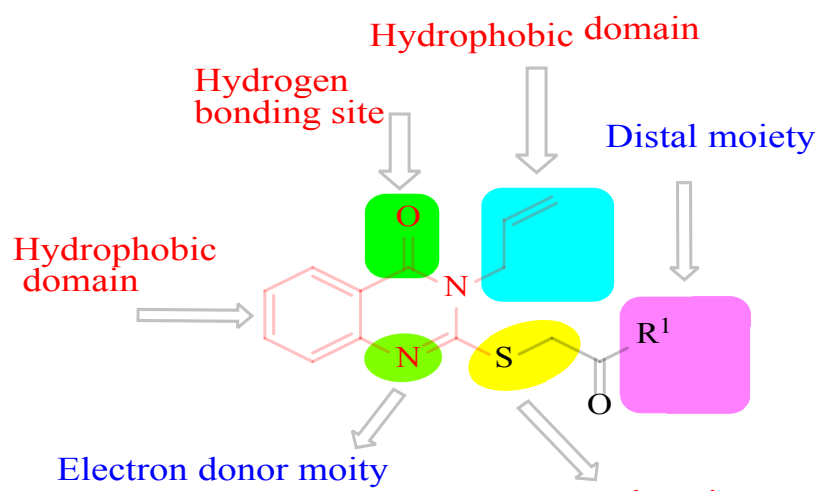

Hydrophobic domain

(4a-e)

Hydrophobic domain

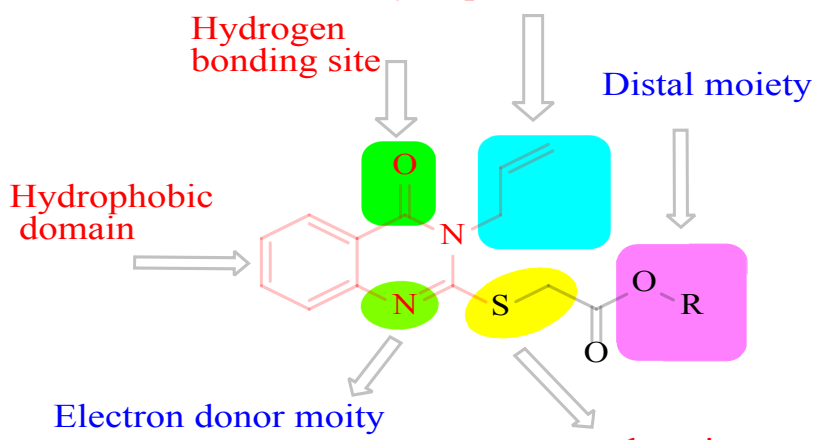

Hydrophobic domain

(6a-e)

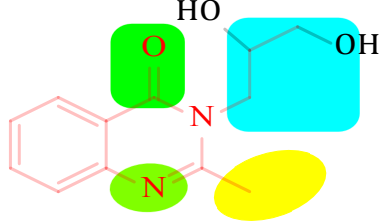

Diproqualone

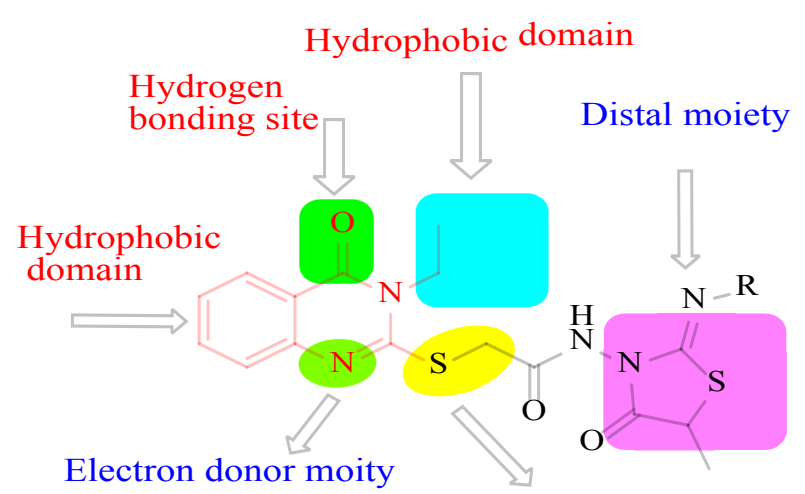

Hydrophobic domain

(B)

Hydrophobic domain

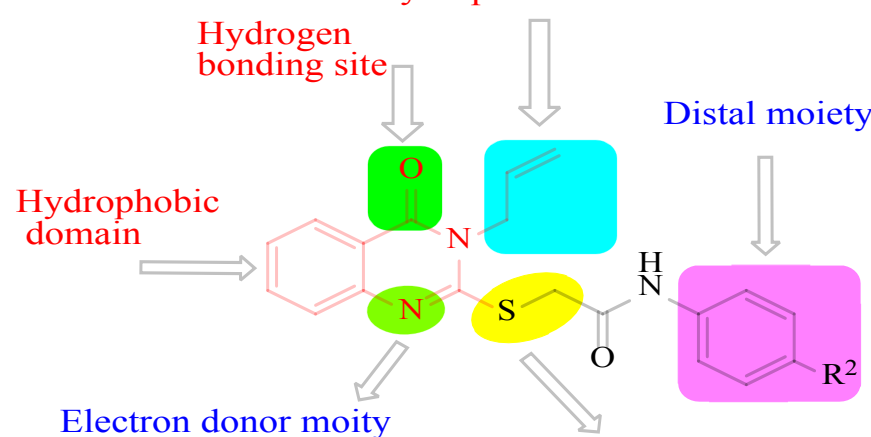

Hydrophobic domain

(5a-d)

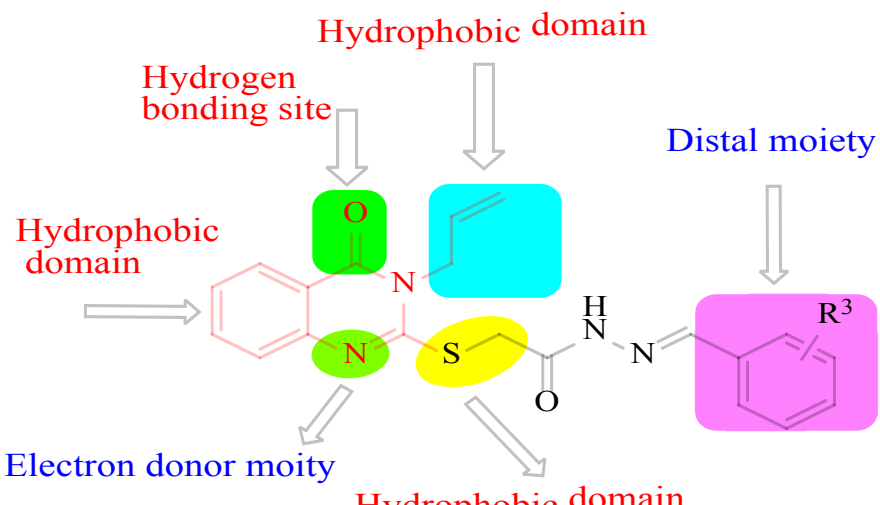

Hydrophobic domain

(8a-d)

Figure 1: Structural similarities and pharmacophoric features of reported and designed quinazolinones (4-8) as anticonvulsants. 
Citation: Abulkhair HS, El-Gamal KM, El-Adl K, Fadl MF (2016) Molecular Docking, Synthesis and Biological Evaluation of Some Novel 2-Substituted3-allyl-4(3H)-quinazolinone Derivatives as Anticonvulsant Agents. Med Chem (Los Angeles) 6: 593-603. doi:10.4172/2161-0444.1000404

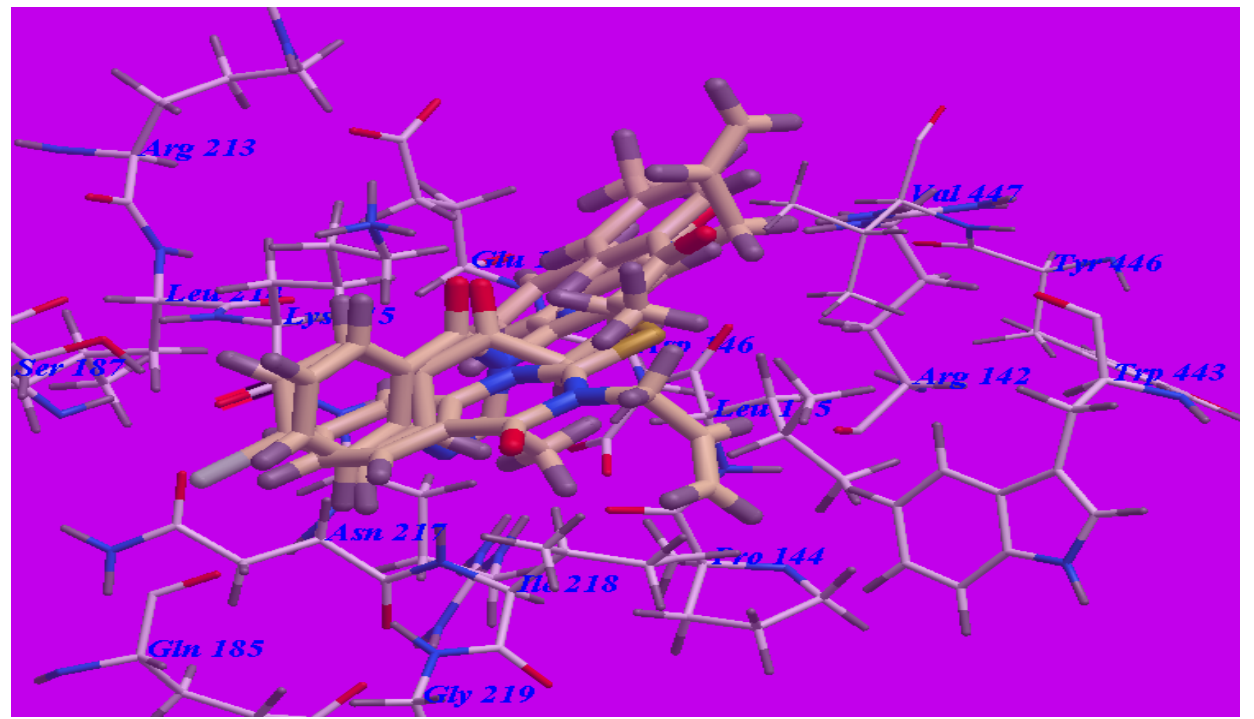

Figure 2: Docking and superimposition of compounds $\mathbf{4}_{\mathrm{c}}, \mathbf{4}_{\mathrm{b}}$ and methaqualone in the vicinity of GABA-A receptor (4COF).

\begin{tabular}{|c|c|c|c|}
\hline Compound & $\Delta \mathbf{G}\left[\mathrm{kcal} \mathrm{mol}{ }^{-1}\right]$ & Compound & $\Delta \mathbf{G}\left[\mathbf{k c a l ~ m o l}^{-1}\right]$ \\
\hline $4 a$ & -69.77 & $6_{b}$ & -72.82 \\
\hline $4_{b}$ & -73.34 & $6_{c}$ & -69.37 \\
\hline $4 \mathrm{c}$ & -74.77 & $6_{d}$ & -69.52 \\
\hline $4_{d}$ & -73.32 & $6_{e}$ & -69.13 \\
\hline $44_{e}$ & -70.08 & 7 & -70.37 \\
\hline 5 & -66.43 & $8_{a}$ & -67.95 \\
\hline $5_{b}$ & -69.96 & $8_{b}$ & -67.41 \\
\hline 5 c & -67.16 & $8_{c}$ & -67.97 \\
\hline $5_{d}$ & -67.11 & $8_{d}$ & -67.25 \\
\hline 6 & -68.12 & Methaqualone & -50.69 \\
\hline
\end{tabular}

Table 1: The calculated $\Delta \mathrm{G}$ (free energy of binding) and binding affinities for the ligands.

Tryptophan443, Tyrosine446 and Valine447. The methyl group at position-2 was oriented in the hydrophobic cleft formed by Proline144, Leucine145, Arginine216, Asparagine217 and Isoleucine218.

The proposed binding mode of compound $\mathbf{4}_{\mathrm{c}}$ (affinity value of $-74.77 \mathrm{kcal} / \mathrm{mol}$ and $4 \mathrm{H}$-bonds) is virtually the same as that of methaqualone (Figure 4) where the linker carbonyl group formed 2 hydrogen bonds with Lysine215 (-NH groups) with distances of $1.82 \AA$ and $3.15 \AA$. The carbonyl group at position- 4 formed 2 hydrogen bonds with Arginine142 (-NH groups) with distances of $2.02 \AA$ and $2.95 \AA$. The quinazolinone moiety occupied the hydrophobic pocket formed by Arginine142, Leucine145, Aspartate146, Glutamate147, Lysine215 and Tyrosine446. The allyl group at position-2 was oriented in the hydrophobic cleft formed by Arginine142, Leucine145, Tyrosine446 and Valine447. The $\mathrm{SCH}_{2}$ linker occupied the hydrophobic cleft formed by Leucine145 and Tryptophan443. The distal phenyl ring linked to position-3 was oriented in the hydrophobic pocket formed by Serine187, Lysine215, Asparagine217, Isoleucine 218 and Glycine219. These interactions of compound $\mathbf{4}$ may explain the highest binding free energy and anticonvulsant activity.

Moreover, the proposed binding mode of compounds $\mathbf{4}_{\mathrm{b}}$ (affinity value of $-73.34 \mathrm{kcal} / \mathrm{mol}$ and $4 \mathrm{H}$-bonds) is virtually the same as that of 4 c (Figure 5) where the linker carbonyl group was stabilized by formation

of 2 hydrogen bonds with Lysine215 (-NH groups) with a distances of $1.64 \AA$ and $1.94 \AA$. The carbonyl group at position- 4 formed one hydrogen bond with Isoleucine 218 (-NH group) with a distance of 2.16 $\AA$ while the N-1 atom formed another hydrogen bond with Lysine 215 (-NH group) with a distance of $2.37 \AA$. The quinazolinone moiety occupied the hydrophobic pocket formed by Serine187, Lysine215, Asparagine217 and Isoleucine218. The allyl group at position-2 was oriented in the hydrophobic cleft formed by Arginine142, Leucine145, Aspartate146, Glutamate147, Lysine215, Asparagine217 and Isoleucine218. The $\mathrm{SCH}_{2}$ linker occupied the hydrophobic cleft formed by Leucine145, Tryptophan443 and Valine447. The distal phenyl ring linked to position-3 was oriented in the hydrophobic pocket formed by Arginine142, Leucine145, Aspartate146, Glutamate147, and Valine447. These interactions of compound $\mathbf{4}_{\mathrm{b}}$ may explain the high binding free energy and anticonvulsant activity.

\section{Chemistry}

In the present work, novel quinazolinone derivatives were prepared as shown in Scheme 1. 3-Allyl-2,3-dihydro-2-thioxoquinazolin-4(1H)one 3 was prepared by fusing anthranilic acid with allylisothiocyanate. The ketone derivatives $4_{\text {a-e }}$ were obtained through reaction of 3 with chloroacetone and/or the appropriate bromoacetophenone. Condensation of $\mathbf{3}$ with $\alpha$-chloroactanilide derivatives yielded the corresponding amides $\mathbf{5}_{\mathrm{a}-\mathrm{d}}$. Ester derivatives $\mathbf{6}_{\mathrm{a}-\mathrm{e}}$ were obtained through condensation of 3 with alkyl $\alpha$-chloroacetates. Treatment of $6_{b}$ with hydrazine hydrate yielded the corresponding hydrazide 7 . Furthermore, Schiff bases $\mathbf{8}_{\text {a-d }}$ were achieved through condensation of the acid hydrazide derivative 7 with the appropriate aromatic aldehydes.

\section{Anticonvulsant activity}

The anticonvulsant activity of the target compounds was evaluated by the use of standard techniques [19], while the acute neurotoxicity was done according to the method described by Dunham and Miya [20]. The preliminary screening was performed at $0.5 \mathrm{mmol} / \mathrm{kg}$ of the selected synthesized compounds by chemical induction of the convulsions using of pentylenetetrazole (PTZ) and electrical induction of convulsions using maximal electroshock (MES) models of seizures [21]. The MES model seems highly predictive of the ability of those 
Citation: Abulkhair HS, El-Gamal KM, El-Adl K, Fadl MF (2016) Molecular Docking, Synthesis and Biological Evaluation of Some Novel 2-Substituted3-allyl-4(3H)-quinazolinone Derivatives as Anticonvulsant Agents. Med Chem (Los Angeles) 6: 593-603. doi:10.4172/2161-0444.1000404

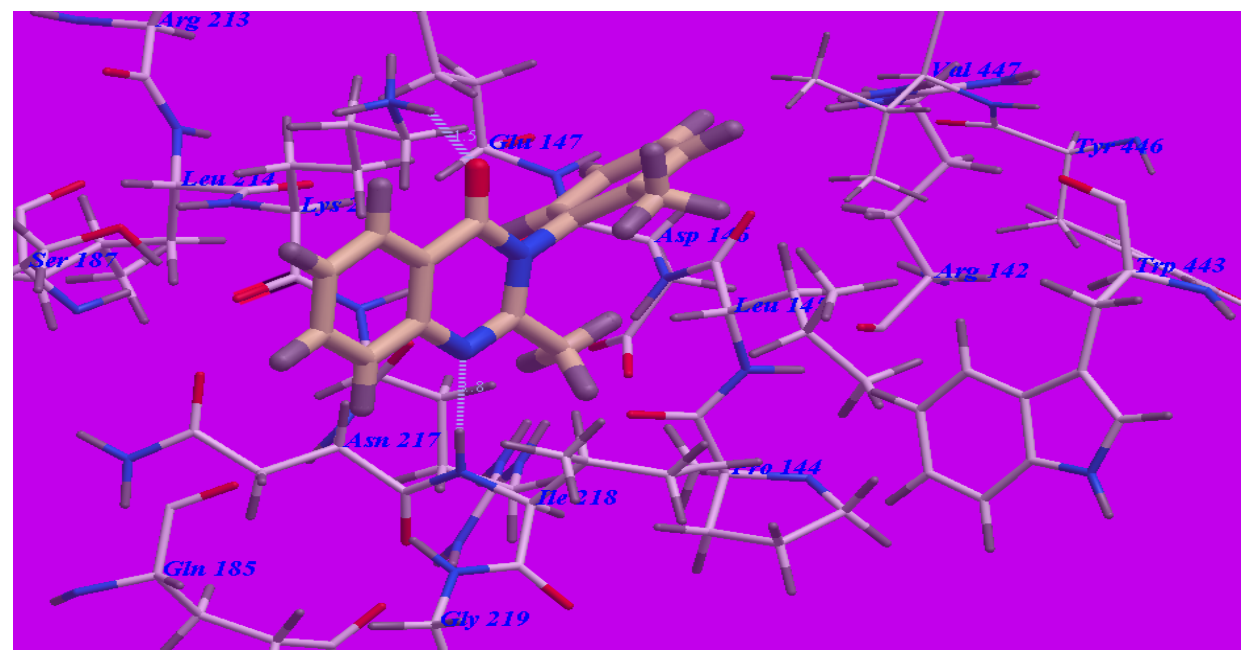

Figure 3: Predicted binding mode for methaqualone with GABA-A receptor (4COF). H-bonds are indicated by dotted lines.

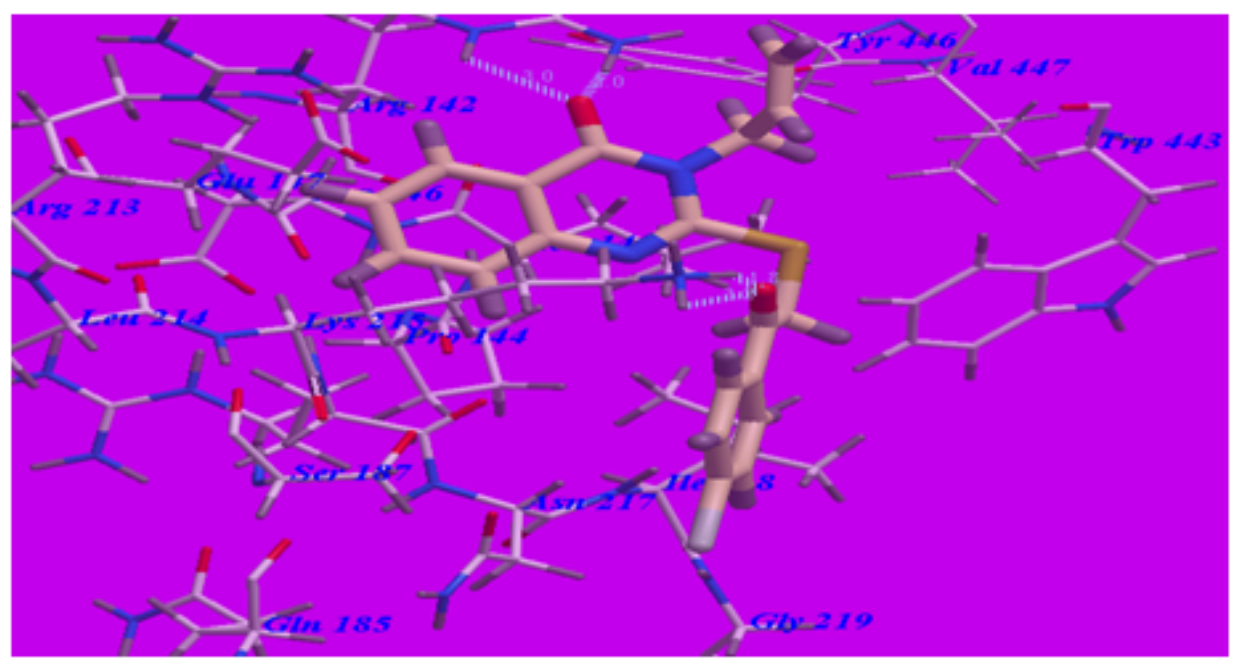

Figure 4: Predicted binding mode for compound $\mathbf{4}_{\mathrm{c}}$ with GABA-A receptor.

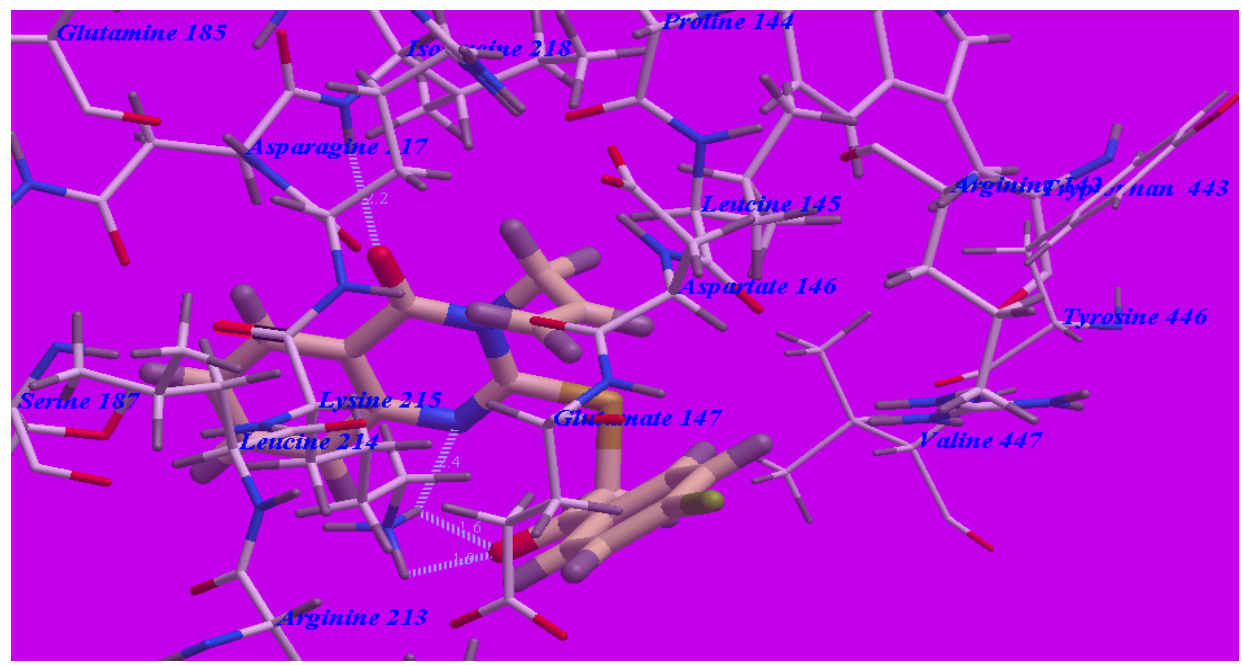

Figure 5: Predicted binding mode for compound $\mathbf{4}_{b}$ with GABA-A receptor. 
Citation: Abulkhair HS, El-Gamal KM, El-Adl K, Fadl MF (2016) Molecular Docking, Synthesis and Biological Evaluation of Some Novel 2-Substituted3-allyl-4(3H)-quinazolinone Derivatives as Anticonvulsant Agents. Med Chem (Los Angeles) 6: 593-603. doi:10.4172/2161-0444.1000404

anticonvulsants to protect against generalized tonic-clonic seizures. The scPTZ model has been proven to be a good predictor of clinical efficacy against generalized spike-wave epilepsies which are known as the absence seizures. Thus, the MES and scPTZ screens have become the most widely employed seizure models for initial identification of candidate anticonvulsants [22]. The initial anticonvulsant evaluation showed that all of the target compounds were active against PTZinduced convulsion in mice at a dose of $0.5 \mathrm{mmol} / \mathrm{kg}$, among which compounds $4,4,4,4_{\mathrm{d}}, 5_{\mathrm{b}}, 6_{\mathrm{b}}$ and 8 showed $100 \%$ protection, compounds 4 and $6_{d}$ showed $83 \%$, compounds $4,5,6,8_{b}$ and 8 showed $66 \%$ protection while compounds 5 and $5_{d}$ showed $50 \%$ protection. For the MES test, compounds 4 , 4 and 4 showed $100 \%$ protection, compounds $5,5,6,6,6$ and 8 showed $66 \%$ protection, compounds $4_{e}$ and $8_{b}$ showed $50 \%$ protection while compounds $4,5,5,6$ and 8 presented $33 \%$ protection.

From the result of preliminary screening (Table 2), the most active compounds $4_{b}, 4_{c}$ and $4_{d}$ were subjected to further investigations in mice i.p. at different doses for quantification of their anticonvulsant activity which indicated by the effective dose causing protection for $50 \%$ of mice $\left(\mathrm{ED}_{50}\right)$ and neurotoxicity which indicated by median toxic dose producing minimal neurological toxicity in $50 \%$ of mice $\left(\mathrm{TD}_{50}\right)$. As demonstrated in Table 3, the selected compounds $4 \mathrm{~b}, 4 \mathrm{c}$ and 4 exhibited anticonvulsant activity against PTZ-induced seizure

\begin{tabular}{|c|c|c|c|}
\hline Compound & $\begin{array}{c}\text { PTZ } \\
\text { (Protection \%) }\end{array}$ & $\begin{array}{c}\text { MES } \\
\text { (Protection \%) }\end{array}$ & C log P \\
\hline $\mathbf{4}_{\mathrm{a}}$ & 66 & 33 & 2.16 \\
\hline $\mathbf{4}_{\mathrm{b}}$ & 100 & 100 & 4.44 \\
\hline $\mathbf{4}_{\mathrm{c}}$ & 100 & 100 & 4.57 \\
\hline $\mathbf{4}_{\mathrm{d}}$ & 100 & 100 & 4.21 \\
\hline $\mathbf{4}_{\mathrm{e}}$ & 83 & 50 & 3.72 \\
\hline $\mathbf{5}_{\mathrm{a}}$ & 50 & 33 & 3.42 \\
\hline $\mathbf{5}_{\mathrm{b}}$ & 100 & 66 & 4.23 \\
\hline $\mathbf{5}_{\mathrm{c}}$ & 66 & 66 & 3.58 \\
\hline $\mathbf{5}_{\mathrm{d}}$ & 50 & 33 & 3.48 \\
\hline $\mathbf{6}_{\mathrm{b}}$ & 100 & 66 & 2.85 \\
\hline $\mathbf{6}_{\mathrm{c}}$ & 66 & 33 & 3.36 \\
\hline $\mathbf{6}_{\mathrm{d}}$ & 83 & 66 & 3.22 \\
\hline $\mathbf{8}_{\mathbf{a}}$ & 100 & 66 & 4.37 \\
\hline $\mathbf{8}_{\mathrm{b}}$ & 66 & 50 & 3.85 \\
\hline $\mathbf{8}_{\mathrm{c}}$ & 66 & 33 & 4.95 \\
\hline Methaqualone & 100 & 100 & 3.03 \\
\hline Valproate & 100 & 100 & 2.80 \\
\hline
\end{tabular}

PTZ: Pentylenetetrazol; MES: Maximal electroshock test.

Table 2: Preliminary anticonvulsant activity of the new synthesized compounds $(0.5 \mathrm{mmol} / \mathrm{kg})$, valproate $(1.8 \mathrm{mmol} / \mathrm{kg})$ and methaqualone $(0.8 \mathrm{mmol} / \mathrm{kg})$.

\begin{tabular}{|c|c|c|c|c|c|}
\hline Compound & $\begin{array}{c}E D_{50}(\mathrm{mg} / \\
\mathrm{kg})\end{array}$ & $\begin{array}{c}\mathrm{TD}_{50}(\mathrm{mg} / \\
\mathrm{kg})\end{array}$ & $\begin{array}{c}\mathrm{LD}_{50}(\mathrm{mg} / \\
\mathrm{kg})\end{array}$ & $\begin{array}{c}\text { Therapeutic } \\
\text { index (TI) }\end{array}$ & $\begin{array}{l}\text { Protective } \\
\text { index (PI) }\end{array}$ \\
\hline $4 b$ & 62 & 175 & 325 & 5.24 & 2.82 \\
\hline $4_{c}$ & 50 & 150 & 375 & 7.50 & 3.00 \\
\hline $4_{d}$ & 75 & 150 & 325 & 4.33 & 2.00 \\
\hline Methaqualone & 200 & 400 & 500 & 2.50 & 2.00 \\
\hline Valproate & 300 & 450 & 500 & 1.66 & 1.50 \\
\hline
\end{tabular}

$\mathrm{ED}_{50}$ : median effective dose providing anticonvulsant protection in $50 \%$ of mice against pentylenetetrazole (PTZ) induced seizures.

$\mathrm{TD}_{50}$ : median toxic dose producing minimal neurological toxicity in $50 \%$ of mice.

$\mathrm{LD}_{50}$ : median lethal dose that causes $50 \%$ mortality in mice.

Therapeutic index: $\mathrm{LD}_{50} / \mathrm{ED}_{50}$

Protective index: $\mathrm{TD}_{50} / \mathrm{ED}_{50}$.

Table 3: $\mathrm{ED}_{50}, \mathrm{TD}_{50}, \mathrm{LD}_{50}$, therapeutic index $(\mathrm{TI})$ and protective index $(\mathrm{PI})$ for the most active compounds $4_{b}, 4_{c}$ and $4_{d}$ compared to the reference drugs methaqualone and Valproate. with $\left(\mathrm{ED}_{50}\right)$ values of 62,50 and $75 \mathrm{mg} / \mathrm{kg}$, respectively. Methaqualone and valproate were used as reference drugs produced $\left(\mathrm{ED}_{50}\right)$ values of 200 and $300 \mathrm{mg} / \mathrm{kg}$, respectively. The $\left(\mathrm{ED}_{50}\right)$ values of the selected compounds were found to be smaller than the reference anticonvulsant drugs at molar doses. The protective index $(\mathrm{PI})=\left(\mathrm{TD}_{50} / \mathrm{ED}_{50}\right)$ is defined to be an index representing the margin of safety and tolerability between anticonvulsant doses and doses of anticonvulsant drugs exerting acute adverse effects like sedation, motor coordination impairment, ataxia, or any other neurotoxic manifestations [23]. The most potent compounds $4_{\mathrm{b}}, 4_{\mathrm{c}}$ and $4_{\mathrm{d}}$ had $\left(\mathrm{TD}_{50}\right)$ values of 175,150 and $150 \mathrm{mg} /$ $\mathrm{kg}$ respectively. These values revealed that these agents exerted low neurological disorders. From the results of $\mathrm{ED}_{50}$ and $\mathrm{TD}_{50}$, compounds $4_{b}, 4_{c}$ and $4_{d}$ had (PI) values of $2.82,3.00$, and 2.00 , which means that compounds $4,4_{c}$ and 4 had higher (PI) values than or equal to the reference drugs as compared to 2.0 for methaqualone and 1.50 for valproate as shown in Table 3 . It is obvious that the protective index values for these selected compounds revealed a difference between the doses producing neurotoxic action $\left(\mathrm{TD}_{50}\right)$ and those exerting anti-PTZ $\left(\mathrm{ED}_{50}\right)$ actions in mice.

The present results are implying high safety margin of the synthesized compounds when compared with the reference drugs. The therapeutic index $(\mathrm{TI})=\left(\mathrm{LD}_{50} / \mathrm{ED}_{50}\right)$ is a comparison of the amount of a therapeutic agent that causes the therapeutic effect to the amount that causes death in mice [13]. Compounds $4_{b}, 4_{c}$ and $4_{d}$ had $\left(\mathrm{LD}_{50}\right)$ values of 325,375 , and $325 \mathrm{mg} / \mathrm{kg}$ with (TI) values of 5.24, 7.50 and 4.33 respectively. It is worthwhile to note that (TI) values of the most potent compounds $4_{b}, 4_{c}$ and $4_{d}$ were found to be higher than that of the reference anticonvulsant drugs as shown in Table 3 . The results of the anticonvulsion screening methods in the present study showed that some new quinazolinones were effective in controlling the seizures induced by PTZ and MES. It has been reported that the convulsants induce seizures by inhibiting $\gamma$-aminobutyric acid (GABA) neurotransmission (such as PTZ), GABA -antagonist or directly antagonizes the inhibitory spinal reflexes of glycine (such as strychnine) [24-26]. Generally, in the MES test, one can determine the anti-seizure effects of agents or drugs that suppress tonic-clonic seizures by suggesting that those compounds possess the ability to prevent the spread of seizure discharge throughout neuronal tissues and to raise seizure threshold [20]. On account of their partial effectiveness, it is difficult to report that our synthesized compounds as having anticonvulsant effects via influencing glycine neurotransmission. However, most of our new compounds can control the seizures induced by PTZ and MES this might suggest that these compounds exhibit a broad spectrum of anticonvulsant activities in animal models of partial and generalized epilepsy via GABA activation. In addition, more detailed study on the GABA pathways and the neurotransmitter levels might be interesting and might provide more insights for the anticonvulsant effects of these new 4(3H)-quinazolines which will be considered extensively in our future study. However, at present some of the new synthesized compounds have relatively potent anticonvulsant effects combined with relatively low neurotoxicity. The high lipid solubility of the target compounds might account for their good activity.

Structure activity relationship (SAR) based on the obtained results indicated that, modifications of methaqualone at positions- 2 and 3 by replacement of methyl group with different substituted distal moieties linked to position-2 through $\mathrm{SCH}_{2} \mathrm{CO}, \mathrm{SCH}_{2} \mathrm{CONH}, \mathrm{SCH}_{2} \mathrm{COO}$ and/or $\mathrm{SCH}_{2} \mathrm{CONHN}$ linkers and replacement of the phenyl group at position-3 of quinazolin-4 $(3 \mathrm{H})$-one by allyl one led to significant improvement of anticonvulsant activity. Different substitution on the position-2 from $4(3 \mathrm{H})$-quinazolinone ring exerted varied anticonvulsant activity. The 
Citation: Abulkhair HS, El-Gamal KM, El-Adl K, Fadl MF (2016) Molecular Docking, Synthesis and Biological Evaluation of Some Novel 2-Substituted3-allyl-4(3H)-quinazolinone Derivatives as Anticonvulsant Agents. Med Chem (Los Angeles) 6: 593-603. doi:10.4172/2161-0444.1000404

lipophilicity and electronic nature of the substituent group led to a significant variation in anticonvulsant activity. It is clear that: $\mathrm{SCH}_{2} \mathrm{CO}$ linker enhanced anticonvulsant activity more than the other linkers and the presence of mono electron withdrawing group exhibited higher activity when compared to unsubstituted, disubstituted and/or electron donating group.

From the data shown above, we can divide these tested compounds into four groups. The first group is the ketone group $\mathbf{4}_{\mathrm{a}-\mathrm{e}}$ which exhibited the highest anticonvulsant activities. Among this group, the phenyl ring enhanced the anticonvulsant activity. The presence of hydrophobic electron withdrawing group (e.g., $\mathrm{Br}$ and $\mathrm{Cl}$ ) at the phenyl ring enhanced the activity when compared to hydrophobic electron donating group $\left(\mathrm{CH}_{3}\right)$ and hydrophilic electron withdrawing group $\left(\mathrm{NO}_{2}\right)$ in the phenyl ring and the order of analogs activity was bromo derivative $\left(\mathbf{4}_{\mathrm{c}}\right)>$ chloro analog $\left(\mathbf{4}_{\mathrm{b}}\right)>$ methyl analog $\left(\mathbf{4}_{\mathrm{d}}\right)>\mathrm{NO}_{2}$ analog $\left(\mathbf{4}_{\mathrm{e}}\right)$. The amide group $\mathbf{5}_{\mathrm{a}-\mathrm{d}}$ is the second group. Among this group, the presence of electron withdrawing group (e.g., $\mathrm{Br}$ and $\mathrm{F}$ ) at the phenyl ring enhanced the activity when compared to electron donating group $\left(\mathrm{OCH}_{3}\right)$ and unsubstituted phenyl ring. The order of analogs activity was bromo derivative $\left(\mathbf{5}_{\mathrm{b}}\right)>$ fluoro analog $\left(\mathbf{5}_{\mathrm{c}}\right)>$ methoxy analog $\left(\mathbf{5}_{\mathrm{d}}\right)$ and unsubstituted analog $\left(\mathbf{5}_{\mathrm{a}}\right)$. The third group is the ester group $\mathbf{6}_{\mathrm{a}-\mathrm{e}}$. Among this group, the ethyl ester exhibited the highest activity while elongation of the carbon chain (e.g., propyl ester) led to decreased activity and the branched chain (e.g., isopropyl ester) showed higher activity than the non-branched one. The order of analogs activity was ethyl ester $\left(\mathbf{6}_{\mathbf{b}}\right)>$ isopropyl ester $\left(\mathbf{6}_{\mathbf{d}}\right)>$ propyl ester $\left(\mathbf{6}_{\mathrm{c}}\right)$. The Schiff's bases group $\mathbf{8}_{\mathrm{a}-\mathrm{d}}$ is the fourth group. Among this group, the presence of monosubstituted electron withdrawing groups (e.g., $\mathrm{Cl}$ and $\mathrm{F}$ ) at the phenyl ring enhanced the activity when compared to the disubstituted derivatives $\left(2,6-\mathrm{Cl}_{2}\right)$ and the order of analogs activity was monochloro derivative $\left(\mathbf{8}_{\mathrm{a}}\right)>$ fluoro analog $\left(\mathbf{8}_{\mathrm{c}}\right)>$ dichloro analog $\left(\mathbf{8}_{\mathrm{c}}\right)$.

\section{$C \log P$ correlation}

As a trial for interpretation of the correlation between chemical structure of the tested compounds and their biological activity, an attempted correlation of anticonvulsant activity with C Log P data was calculated for the measurement of the lipophilicity factor which could be attributed in their anticonvulsant activity. For antiepileptic agents to be effective, they have to cross the blood brain barrier (BBB) [21]. Crossing the brain is therefore a crucial step in developing effective drug therapies for treatment of neurological disorders. Lipophilic substances are able to permeate into the brain interstitium in a relatively easy way $[1,27]$. Determination of brain-blood partitioning in vitro is difficult, time-consuming, expensive, not always available and not suitable to screen a large collection of new chemicals. Therefore, an alternative method was used based on computerized models. So, the $\mathrm{C}$ $\log \mathrm{P}$ values were calculated for some compounds to reflect the overall lipophilicity of these compounds and compared. It is postulated that a $C \log P$ value of at least 2.0 is required by a specific compound to cross the BBB [28]. The $\mathrm{C} \log \mathrm{P}$ data for all selected anticonvulsant compounds are explained in Table 2 ranging from 2.16 to 4.95 . All the compounds were found to have $\mathrm{C} \log \mathrm{P}$ values above 2 (which required for effective penetration in the brain) and higher than that of methaqualone and valproate except compounds $\mathbf{4}_{\mathrm{a}}$ and $\mathbf{6}_{\mathrm{b}}$.

Within the ketone group $\mathbf{4}_{\mathrm{a}-\mathrm{e}}$ it is worthwhile to note that the $\mathrm{C}$ $\log \mathrm{P}$ values for compounds $\mathbf{4}_{\mathrm{c}^{\mathrm{a}}}, \mathbf{4}_{\mathrm{b}}$ and $\mathbf{4}_{\mathrm{d}}$ which had higher potency were found to be $4.57,4.44$ and 4.21 respectively which might explain the significant variation in their biological activity in correlation with their lipophilicity. Interestingly, the values of $\mathrm{C} \log \mathrm{P}$ for the selected potent compounds agree with their potency levels, compound $\mathbf{4}$ which had the highest activity, had also highest C Log P value (4.57), while compound $\mathbf{4}_{\mathrm{b}}$ which had the medium activity, had medium $\mathrm{C} \log \mathrm{P}$ value (4.44) and compound 4 had lowest $C$ Log $P$ value as its activity (4.21). Compounds 4 and 4 exhibited C Log P value 3.72 and 2.16 respectively in agree with their potency levels. Within the second group $\mathbf{5}_{\text {a-d }}$, all compounds showed anticonvulsant potency correlated with their lipophilicity factor. While in the third group $\mathbf{6}_{\mathbf{b}-\mathbf{d}}$, in spite of compound $\mathbf{6}_{\mathrm{b}}$ exhibited the lowest C Log P value (2.85), it showed the highest anticonvulsant activity and compound $\mathbf{6}_{\mathrm{c}}$ which exhibited the highest $C \log P$ value (3.36), it showed the lowest anticonvulsant activity. All compounds within this group exhibited anticonvulsant potency in the reverse direction of their $\mathrm{C} \log \mathrm{P}$ values. Within the fourth group $\mathbf{8}_{\mathrm{a}-\mathrm{c}}$, only compound $\mathbf{8}_{\mathbf{c}}$ which had the highest $\mathrm{C} \log \mathrm{P}$ value (4.95) than other compounds, showed the lowest potency with no correlation with the lipophilicity factor. In general, it was found that compounds having more $\mathrm{C} \log \mathrm{P}$ values having higher potency as anticonvulsant agents [29-31].

\section{Conclusion}

New derivatives of $4(3 \mathrm{H})$-quinazolinones were synthesized and evaluated for their anticonvulsant activity and acute neurotoxicity in mice. The results of this study demonstrated that compounds belong to the ketone group $4_{\mathrm{a}-\mathrm{e}}$ revealed more activity than the other groups specially, compounds $\mathbf{4}_{\mathrm{c}}, \mathbf{4}_{\mathrm{b}}$ and $\mathbf{4}$ showed better anticonvulsant activity and much lower toxicity compared with the reference drugs. The lipophilicity and electronic nature of the substituent groups selected for this study led to a significant variation in anticonvulsant activity. The obtained results showed that compounds $\mathbf{4}_{c}, \mathbf{4}_{b}$ and $\mathbf{4}_{d}$ could be useful as a template for future design, optimization and investigation to produce more active analogs.

\section{References}

1. Zayed MF, Ahmed HE, Omar ASM, Abdelrahim AS, El-Adl K (2013) Design, synthesis, and biological evaluation studies of novel quinazolinone derivatives as anticonvulsant agents. Med Chem Res 22: 5823-5831.

2. Kumar A (2013) Designing of Hybrid form of Benzothiazole-quinazoline as GABA-A Inhibitor with Anticonvulsant Profile: An in-silico Approach. Am J Pharmacol Sci 1: 116-120.

3. Sahoo BM, Dinda SC, Ravi Kumar BV, Panda JR (2013) Green Synthesis and Evaluation of 3-(Aryl)-2-Thioxo-2, 3-Dihydro-quinazolin-4 (1H)-ones as Novel Anticonvulsant Drugs. Int J Pharm Sci Nanotechnol 6: 2046-2052.

4. Gawad NMA, Georgey HH, Youssef RM, El Sayed NA (2011) Design, synthesis, and anticonvulsant activity of novel quinazolinone analogues. Med Chem Res 20: 1280-1286.

5. Kumar P, Shrivastava B, Pandeya SN, Stables JP (2011) Design, synthesis and potential $6 \mathrm{~Hz}$ psychomotor seizure test activity of some novel 2-(substituted)3-\{[substituted] amino\} quinazolin-4 (3H)-one. Eur J Med Chem 46: 1006-1018.

6. Ilangovan P, Ganguly S, Pandi V (2010) Design and synthesis of nove quinazolinone derivatives as broad spectrum anticonvulsant and antimicrobial agent. J Pharm Res 3: 703-706.

7. Kashaw SK, Kashaw V, Mishra P, Jain NK, Stables JP (2009) Synthesis, anticonvulsant and CNS depressant activity of some new bioactive 1-(4-substituted-phenyl)-3-(4-oxo-2-phenyl/ethyl-4H-quinazolin-3-yl)-urea. Eur J Med Chem 44: 4335-4343.

8. El-Azab AS, EITahir KE (2012) Design, synthesis and anticonvulsant evaluation of novel 8-substituted-4 (3H)-quinazolines. Med Chem Res 21: 3785-3796.

9. Wolfe JF, Rathman TL, Sleevi MC, Campbell JA, Greenwood TD (1990) Synthesis and anticonvulsant activity of some new 2-substituted 3-aryl-4(3H)quinazolinones. J Med Chem 33: 161-166.

10. Jatav V, Mishra P Kashaw S, Stables JP (2008) CNS depressant and anticonvulsant activities of some novel 3-[5-substituted 1, 3, 4-thiadiazole-2-yl] 2-styryl quinazoline-4 (3H)-ones. Eur J Med Chem 43: 1945-1954. 
Citation: Abulkhair HS, El-Gamal KM, El-Adl K, Fadl MF (2016) Molecular Docking, Synthesis and Biological Evaluation of Some Novel 2-Substituted3-allyl-4(3H)-quinazolinone Derivatives as Anticonvulsant Agents. Med Chem (Los Angeles) 6: 593-603. doi:10.4172/2161-0444.1000404

11. Johnston GAR (1996) GABAA receptor pharmacology. Pharmacol Ther 69: 173-198.

12. Ibrahim MK, El-Adl K, Al-Karmalawy AA (2015) Design, synthesis, molecular docking and anticonvulsant evaluation of novel 6-iodo-2-phenyl-3-substitutedquinazolin-4 (3H)-ones. Bulletin of Faculty of Pharmacy, Cairo University 53: 101-116.

13. Zappala M, Grasso S, Micale N, Zuccala G, Menniti FS, et al. (2003) 1-Aryl-6, 7-methylenedioxy-3H-quinazolin-4-ones as anticonvulsant agents. Bioorg Med Chem Lett 13: 4427-4430.

14. Bayoumi A, Ghiaty A, El-Morsy A, Abul-Khair H, Hassan MH, et al. (2012) Synthesis and evaluation of some new 1, 2, 4-triazolo (4, 3-a) quinoxalin-4-5Hone derivatives as AMPA receptor antagonists. Bulletin of Faculty of Pharmacy, Cairo University 50: 141-146.

15. Topliss JG (1977) A manual method for applying the Hansch approach to drug design. J Med Chem 20: 463-469.

16. Gürsoy A, Terzioğlu N (2005) Synthesis and isolation of new regioisomeric 4-thiazolidinones and their anticonvulsant activity. Turk J Chem 29: 247-254.

17. Englert L, Biela A, Zayed M, Heine A, Hangauer D, et al. (2010) Displacement of disordered water molecules from hydrophobic pocket creates enthalpic signature: Binding of phosphonamidate to the $S 1^{1}$-pocket of thermolysin. Biochim Biophys Acta 1800: 1192-1202.

18. Baum B, Mohamed M, Zayed M, Gerlach C, Heine A, et al. (2009) More than a simple lipophilic contact: a detailed thermodynamic analysis of nonbasic residues in the s1 pocket of thrombin. $\mathrm{J}$ Mol Biol 390: 56-69.

19. Yogeeswari $P$, Sriram D, Thirumurugan R, Raghavendran JV, Sudhan K, et al. (2005) Discovery of N-(2,6-dimethylphenyl)-substituted semicarbazones as anticonvulsants: hybrid pharmacophore-based design. J Med Chem 48: 6202-6211.

20. Dunham NW, Miya TS (1957) A note on a simple apparatus for detecting neurological deficit in rats and mice. J Am Pharm Assoc 46: 208-209.

21. Crivori P, Cruciani G, CarruptPA, Testa B (2000) Predicting blood-brain barrier permeation from three-dimensional molecular structure. J Med Chem 43: 2204-2216.
22. Rogawski MA (2006) Point-counterpoint: Do interictal spikes trigger seizures or protect against them? Epilepsy Curr 6: 197-198.

23. Löscher W, Nolting B (1991) The role of technical, biological and pharmacological factors in the laboratory evaluation of anticonvulsant drugs. IV. Protective indices. Epilepsy Res 9: 1-10.

24. Okada R, Negishi N, Nagaya H (1989) The role of the nigrotegmental GABAergic pathway in the propagation of pentylenetetrazol-induced seizures. Brain Res 480: 383-387.

25. Lacoste L, Bartolucci S, Lapointe J (1988) Pentylenetetrazole inhibits glutamate dehydrogenase and aspartate aminotransferase, and stimulates GABA aminotransferase in homogenates from rat cerebral cortex. Can J Physiol Pharmacol 66: 1135-1138.

26. Olsen RW (1981) GABA-benzodiazepine-barbiturate receptor interactions. J Neurochem 37: 1-13

27. Bhaduri AP, Khanna NM, Dhar ML (1964) Potential anticonvulsants: Synthesis of 2,3-substituted 4-quinazolones and quinazolo-4-thiones. Ind J Chem 2: 159-161.

28. Shank RP, Gardocki JF, Streeter AJ, Maryanoff BE (2000) An overview of the preclinical aspects of topiramate: pharmacology, pharmacokinetics, and mechanism of action. Epilepsia 41: 3-9.

29. Sachdev HS, Dhami KS, Atwal MS (1961) Thiopegan derivatives-XVI: Synthesis of 10: 11-thiopegan derivatives containing a saturated thiazole moiety and of their related compounds. Tetrahedron 14: 304-309.

30. Vogel GH (2008) Drug Discovery and Evaluation: Pharmacological Assays. 3rd edn. Springer-Verlag, New York, USA, pp: 692-693.

31. Woodbury LA, Davenport VD (1952) Design and use of a new electroshock seizure apparatus, and analysis of factors altering seizure threshold and pattern. Arch Int Pharmacodyn Ther 92: 97-107. 